\title{
Monocyte NOTCH2 expression predicts IFN- $\beta$ immunogenicity in multiple sclerosis patients
}

\author{
Marsilio Adriani, ${ }^{1}$ Petra Nytrova, ${ }^{2}$ Cyprien Mbogning, ${ }^{3}$ Signe Hässler, ${ }^{3}$ Karel Medek, ${ }^{2}$ \\ Poul Erik H. Jensen, ${ }^{4}$ Paul Creeke, ${ }^{5}$ Clemens Warnke, ${ }^{6,7}$ Kathleen Ingenhoven, ${ }^{6}$ Bernhard Hemmer, ${ }^{8}$ \\ Claudia Sievers, ${ }^{9}$ Raija L.P. Lindberg Casser, ${ }^{9}$ Nicolas Fissolo, ${ }^{10}$ Florian Deisenhammer, ${ }^{11}$ \\ Zsolt Bocskei, ${ }^{12}$ Vincent Mikol, ${ }^{12}$ Anna Fogdell-Hahn, ${ }^{13}$ Eva Kubala Havrdova, ${ }^{2}$ \\ Philippe Broët, ${ }^{3,14}$ Pierre Dönnes, ${ }^{15}$ Claudia Mauri, ${ }^{1}$ Elizabeth C. Jury', \\ and The ABIRISK Consortium ${ }^{16}$ \\ 'Department of Rheumatology, University College Hospital, London, United Kingdom. ²Department of Neurology \\ and Center for Clinical Neuroscience, First Faculty of Medicine, Charles University and General University Hospital in \\ Prague, Czech Republic. ${ }^{3}$ CESP, Fac. De Médecine-Univ. Paris-Sud, Fac. De Médecine-UVSQ, INSERM, Université Paris- \\ Saclay, Villejuif, France. ${ }^{4}$ Neuroimmunology Laboratory, DMSC, Department of Neurology, Rigshospitalet, Region H, \\ Copenhagen, Denmark. ${ }^{5}$ Neuroimmunology Unit, Centre for Neuroscience and Trauma, Blizard Institute, Queen Mary \\ University of London, London, United Kingdom. ${ }^{6}$ Department of Neurology, Medical Faculty, Research Group for Clinical \\ and Experimental Neuroimmunology, Heinrich-Heine-University, Düsseldorf, Germany. University Hospital Koeln, \\ Deptartment of Neurology, Koeln, Germany. ${ }^{8}$ Klinikum rechts der Isar, Department of Neurology, School of Medicine, \\ Technical University of Munich, Munich, Germany. ' ${ }^{9}$ Laboratory of Clinical Neuroimmunology, Departments of Biomedicine \\ and Clinical Research, University Hospital Basel and University of Basel, Basel, Switzerland. ${ }^{10}$ Centre d'Esclerosi Múltiple \\ de Catalunya (Cemcat), Hospital Universitari Vall d'Hebron, Barcelona, Spain. "Clinical Department of Neurology, Innsbruck \\ Medical University, Innsbruck, Austria. ${ }^{12}$ Translational Sciences Unit, Sanofi R\&D, 91385 Chilly-Mazarin, Paris, France. \\ ${ }^{13}$ Karolinska Institutet, Department of Clinical Neuroscience, Center for Molecular Medicine (CMM), Karolinska University \\ Hospital, Sweden. ${ }^{14}$ Assistance Publique - Hôpitaux de Paris, Hôpital Paul Brousse, Villejuif, France. ${ }^{15}$ Scicross AB, Skövde, \\ Sweden. ${ }^{16}$ See Supplemental Acknowledgments for ABIRISK Consortium details.
}

Medimmune, Novartis, Desitin, and F. Hoffmann-La Roche Ltd.; his institution has received research support from Chugai Pharmaceuticals and Hoffmann-La-Roche; he holds part of 2 patents -1 for the detection of antibodies and $\mathrm{T}$ cells against KIR4. 1 in a subpopulation of MS patients and 1 for genetic determinants of neutralizing antibodies to interferon $\beta$. EKH has received honoraria/research support from Actelion, Biogen, Czech Ministry of Education project PROGRES Q27/LF1, Sanofi Cenzyme, Merck Serono, Novartis, Roche, and Teva.

License: This work is licensed under the Creative Commons Attribution 4.0 International License. To view a copy of this license, visit http:// creativecommons.org/licenses/ by/4.0\%.

Submitted: December 19, 2017

Accepted: April 24, 2018

Published: June 7, 2018

Reference information: JCI Insight. 2018;3(11):e99274. https://doi.org/10.1172/jici. insight.99274.

Multiple sclerosis (MS) is an autoimmune disease characterized by CNS inflammation leading to demyelination and axonal damage. IFN- $\beta$ is an established treatment for MS; however, up to $30 \%$ of IFN- $\beta$-treated MS patients develop neutralizing antidrug antibodies (nADA), leading to reduced drug bioactivity and efficacy. Mechanisms driving antidrug immunogenicity remain uncertain, and reliable biomarkers to predict immunogenicity development are lacking. Using highthroughput flow cytometry, NOTCH2 expression on $\mathrm{CD}_{14}^{+}$monocytes and increased frequency of proinflammatory monocyte subsets were identified as baseline predictors of nADA development in MS patients treated with IFN- $\beta$. The association of this monocyte profile with nADA development was validated in 2 independent cross-sectional MS patient cohorts and a prospective cohort followed before and after IFN- $\beta$ administration. Reduced monocyte NOTCH2 expression in nADA+ MS patients was associated with NOTCH2 activation measured by increased expression of Notch-responsive genes, polarization of monocytes toward a nonclassical phenotype, and increased proinflammatory IL-6 production. NOTCH2 activation was T cell dependent and was only triggered in the presence of serum from nADA+ patients. Thus, nADA development was driven by a proinflammatory environment that triggered activation of the NOTCH2 signaling pathway prior to first IFN- $\beta$ administration.

\section{Introduction}

Multiple sclerosis (MS) is a multifocal demyelinating disease of the CNS affecting about 2.5 million people worldwide. Although the disease course is highly variable, the majority of patients develop irreversible disability, and MS remains a major cause of neurological disability in young adults (1). Disease etiology remains uncertain but involves a combination of genetic and environmental cues (2) associated with myelin-specific 
A Healthy donor PBMCs

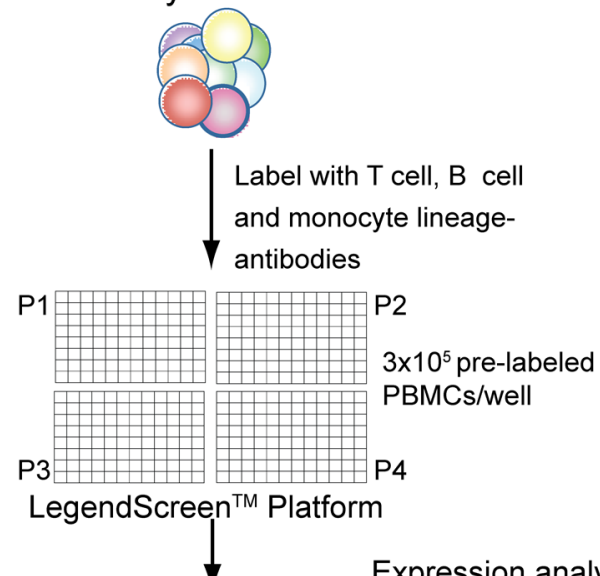

Expression analysis of 332

High-throughput flow cytometry cell surface markers on monocytes, B cells and T cells

C

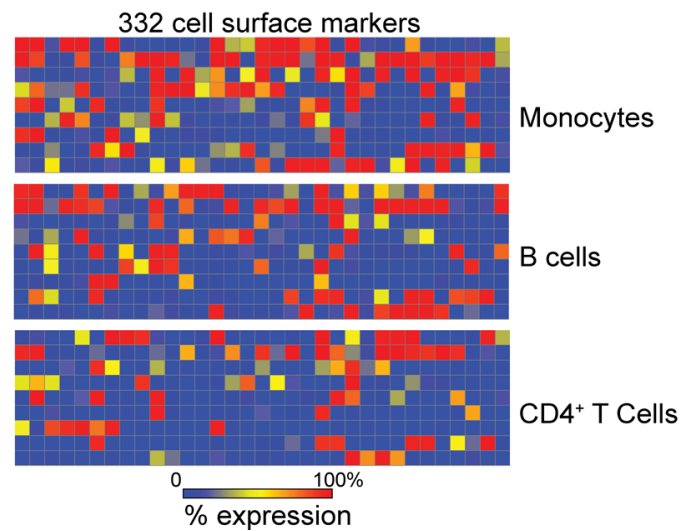

B



Figure 1. High-throughput flow cytometry identified distinct immune signatures in healthy donor lymphocytes and monocytes. (A) Schematic representation of the experimental strategy. A minimum of $3 \times 10^{5}$ peripheral blood mononuclear cells (PBMCs) prelabeled with lineage-specific antibodies to allow T cell, B cell, and monocyte stratification were incubated with the 332 prealiquoted antibodies of the LegendScreen platform in four 96-well plates (P1-P4) and analyzed by high-throughput flow cytometry. (B) Unsupervised hierarchical clustering of the 332 marker expression values (percentage-positive; black, $0 \%$; red, 100\%) in monocytes, B cells, and CD4 ${ }^{+} \mathrm{T}$ cells from $10 \mathrm{HCs}$ showed the unbiased identification of cell subsets. (C) Heatmaps showing the combined expression of the 332 markers on monocytes, B cells, and CD4+ T cells from healthy donors (HCs, $n=10)$, treatment naive ( $n=10)$, and IFN- $\beta$ treated $(n=15)$ patients with multiple sclerosis (discovery cohort, Table 1).

autoreactive $\mathrm{CD}^{+} \mathrm{T}$ cells primed in the periphery and mediating a complex cascade of events leading to progressive neurodegeneration. Recent evidence also implicates wider immune cell involvement from both the adaptive and innate arms of the immune response, including B cells and monocytes (3-7).

In the last 25 years, many disease-modifying drugs (DMD) have become available for patients with MS that aim to prevent, rather than repair, tissue injury (8). IFN- $\beta$ (either IFN- $\beta-1 \mathrm{a}$ or IFN- $\beta-1 b$ ) was one of the first biopharmaceuticals approved for clinical use and has been a first-line DMD in patients with relapsing remitting (RR) MS since 1993 (9-12).

However, all protein biopharmaceuticals, including IFN- $\beta$, can induce an immune response (immunogenicity) associated with the production of antidrug antibodies (ADA). The consequences of immunogenicity range from transient ADA to persistant high-titer ADA with clinical effects (13). Circulating ADA can alter the bioavailability of the drug, either shortening or increasing the elimination half-life of the molecule. Importantly, some ADA have neutralizing activity (neutralizing ADA [nADA] ) - leading to reduced drug efficacy (14) - and, in some cases, can induce autoimmunity to endogenous molecules (15). Between $5 \%$ and $30 \%$ of patients with MS treated with IFN- $\beta$ develop nADA within the first 9-18 months of therapy. In these patients, nADA have been reported to reduce the cellular response to IFN- $\beta$, impacting the therapeutic outcomes of the treatment (16). The formation of ADA can depend on both properties of the product, such as impurity and contamination 
Table 1. Description of MS patient cohorts

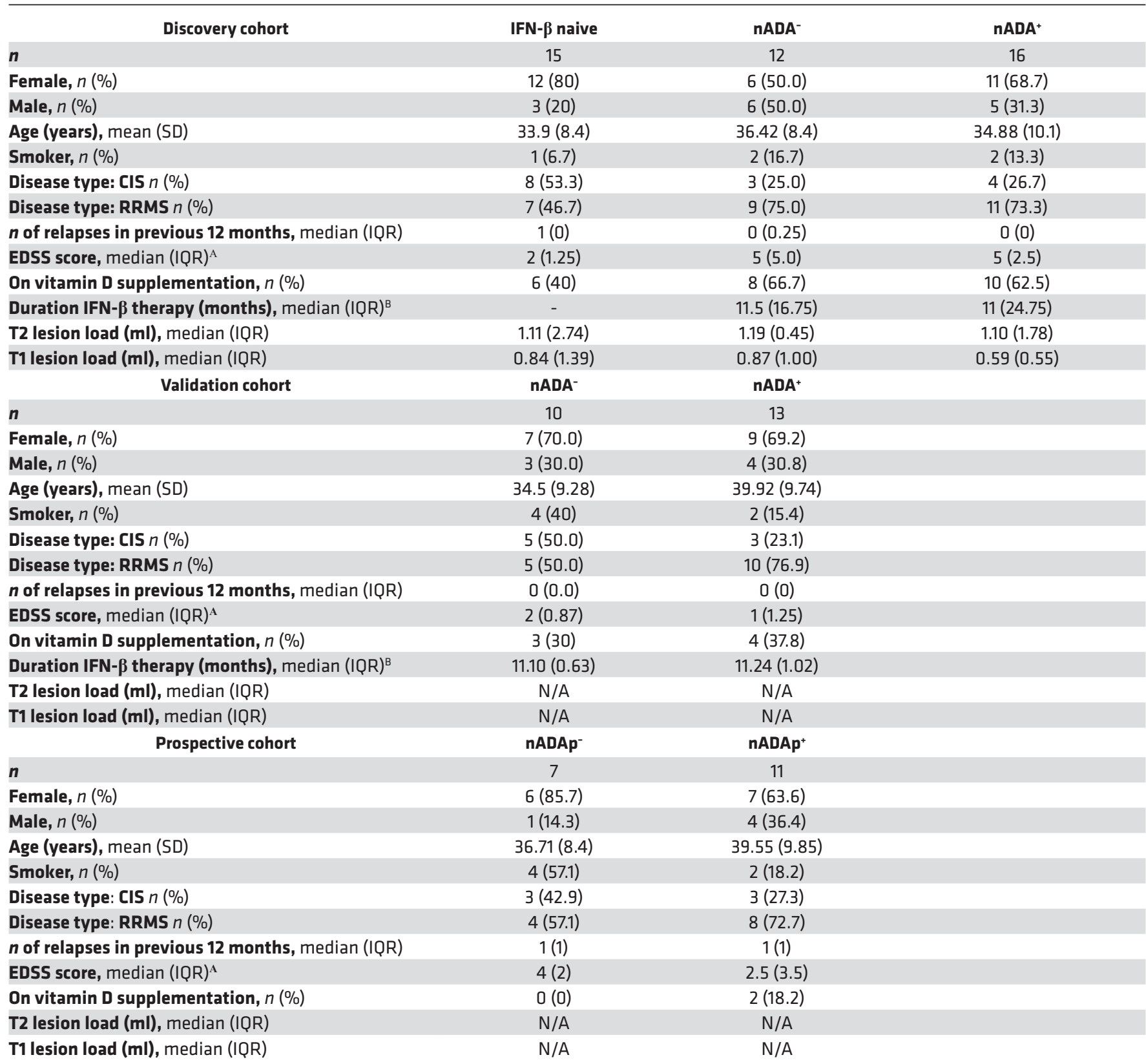

Patients diagnosed with relapsing remitting (RR) MS or clinically isolated syndrome (CIS) according to the revised McDonald criteria (61). Three cohorts were recruited: a cross-sectional discovery cohort, which was either IFN- $\beta$-treatment naive (MS-N) or treated with IFN- $\beta$ for at least 12 months (MS-T); a cross sectional validation cohort, which included MS-T patients on IFN- $\beta$ for at least 12 months; and a prospective cohort, which included MS patients untreated at sampling and follow-up for 12 months before neutralizing antidrug antibody (nADA) determination. Cohorts recruited as part of the ABIRISK consortium (www.abirisk.eu/), with samples collected from patients before their first treatment with IFN- $\beta$ and after 12 months of treatment. In the discovery cohort, a statistical difference was detected between Expanded Disability Status Scale (EDSS) scores from MS-N and MS-T patients (2-tailed $t$ test; $P \leq 0.05$ ). No statistically significant differences were observed in all other clinical/demographic characteristics between the patient groups at baseline (2-tailed $t$ test and $\chi^{2}$ test; $P>0.05$ ). ${ }^{A}$ Scores on the EDSS range from $0-10$, with higher score indicating worse disability. ${ }^{\text {BP }}$ atients received either IFN- $\beta-$ la or IFN- $\beta$-Ib preparations. IQR, interquartile range; N/A, not available.

originating from the manufacturing process (17), and patient-related features, such as specific HLA alleles (18). In addition, differences in immune cell phenotype and activation status could also play an important role during ADA induction. However, no predictive biomarkers are established to improve treatment choices for patients. 
In the present study, high-throughput flow cytometry technology (LegendScreen) and a systems immunology approach was applied to identify potential predictive biomarkers of nADA development in MS patients treated with IFN- $\beta$. Using a systematic analysis framework accounting for the influence of disease progression and IFN- $\beta$ activity, a phenotypic signature associated with nADA development was identified. Importantly, reduced neurogenic locus notch homolog protein-2 (NOTCH2) expression on monocytes was associated with the presence of nADA even before the initiation of IFN- $\beta$ therapy and was instrumental in predicting the eventual development of $\mathrm{nADA}$ in a prospective cohort of MS patients. Moreover, reduced $\mathrm{NOTCH} 2$ expression was associated with increased activation of NOTCH2 signaling and polarization of monocytes toward a proinflammatory phenotype. Thus, we highlight a potentially new tool to assess the response of patients to IFN- $\beta$ therapy and identify potential immunological mechanisms involved in the development of immunogenicity.

\section{Results}

LegendScreen high-throughput flow cytometry identified distinct peripheral blood immune cell signatures. The LegendScreen platform (BioLegend) was validated as a tool to assess the surface signature of immune cell subsets in peripheral blood mononuclear cells (PBMCs) from human healthy control (HC) donors. PBMCs prelabeled with lineage-specific fluorescently conjugated antibodies identifying CD4 ${ }^{+} \mathrm{T}$ cells, B cells, and monocytes were analyzed in tandem with the 332 cell surface markers of the LegendScreen array (Figure 1A and Supplemental Figure 1A; supplemental material available online with this article; https://doi. org/10.1172/jci.insight.99274DS1 for experimental and gating strategy). LegendScreen inter- and intra-assay reproducibility was verified by assessing expression of the 332 markers on PBMCs from the same $\mathrm{HC}$ analyzed on 3 different days. Both the expression of the markers on each cell subset (Supplemental Figure 1B) and the mean fluorescence emissions of plates 1-4 (P1-P4 of the LegendScreen array) from each run (Supplemental Figure 1C) were highly correlated. Also, using known lineage-specific markers, the specificity of the platform for each cell subset (monocytes, B cells, and CD4 ${ }^{+} \mathrm{T}$ cells) was corroborated (Supplemental Figure 1, D-F). Unsupervised hierarchical cluster analysis showed that expression levels of the 332 markers making up the LegendScreen array could discriminate between monocytes, B cells, and $\mathrm{CD}^{+} \mathrm{T}$ cells (Figure 1B). However, many of the 332 markers were negative in all subsets tested. This was confirmed when LegendScreen signatures from HCs and patients with MS were combined (Figure 1C). Therefore, to increase the statistical power of our analyses, the markers consistently negative in all samples tested were excluded (Supplemental Figure 2, A and B, and Methods). Using this approach, 211 monocyte, $170 \mathrm{~B}$ cell, and $125 \mathrm{CD}^{+} \mathrm{T}$ cell markers were retained and used for all future analyses (Supplemental Table 1). Thus, the LegendScreen platform was robust and could be used to stratify human PBMCs into biologically relevant groups and identify proteins differentially associated with specific cell types.

Immunogenicity development in IFN- $\beta$-treated MS patients was associated with a specific monocyte signature. We used the validated LegendScreen platform to investigate immune signatures associated with nADA in 28 MS patients treated with IFN- $\beta$ for at least 12 months (MS-T) who had (nADA ${ }^{+}$) or had not $\left(\mathrm{nDA}^{-}\right)$developed immunogenicity to the drug (Table 1, cross-sectional discovery cohort). A stepwise approach was used. Firstly, differentially expressed markers (DEMs) between nADA ${ }^{+}$and nADA MS-T $^{-}$ patients were identified using 2-tailed unpaired $t$ test analysis and FDR $<0.2$ (Figure 2A and Supplemental Table 2). The majority of DEMs were expressed on monocytes $(n=23)$, with only $3 \mathrm{~B}$ cell markers and no $\mathrm{CD}^{+} \mathrm{T}$ cell markers identified.

Next, a systematic analysis framework was implemented to discriminate between DEMs associated with the presence of nADA alone rather than disease progression and/or the effect of IFN- $\beta$ therapy (Figure $2 \mathrm{~B}$ ). Five DEMs expressed on monocytes (mesenchymal stem cells and neural progenitor cells antigen [MSC-NPC], CD138, TIM3, CD254, and C-type lectin domain family 9-A [CLEC9A]) correlated significantly with MRI evidence of MS disease activity in the brain (measured as change in quantitative MRI [QMRI] T1 and T2 lesion volume in the previous 12 months) (19) and were removed from further analysis (Figure 2, C and D). For all other DEMs, no significant correlations with T1 and T2 lesion volume were observed (Supplemental Table 3).

DEMs associated with IFN- $\beta$ activity were identified by comparing their expression in $\mathrm{nADA}^{+}, \mathrm{nADA}^{-}$ , and IFN- $\beta$-treatment naive (MS-N) MS patients (Table 1, discovery cohort) using 1-way ANOVA. DEMs whose expression was significantly different when comparing $\mathrm{nADA}^{-}$and $\mathrm{MS}-\mathrm{N}$ patients, but not between $\mathrm{nADA}^{+}$and MS-N (Figure 2E, Table 2, and Supplemental Figure 3), were considered to be associated 
A
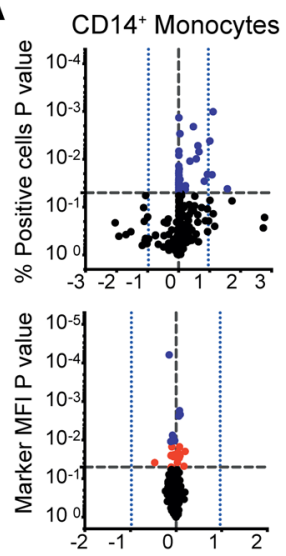

C

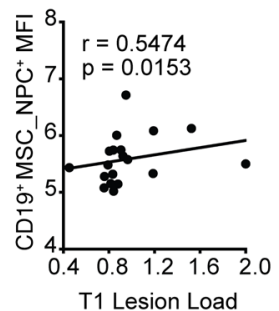

CD19+ $B$ cells
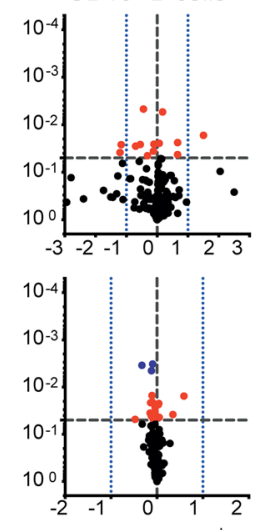

$\mathrm{nADA}^{-}: \mathrm{nADA}^{+}$
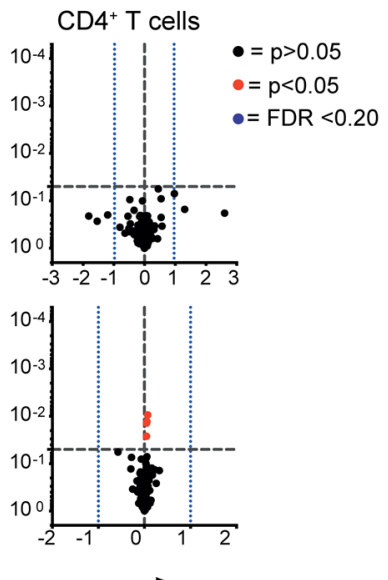

B



\section{D}
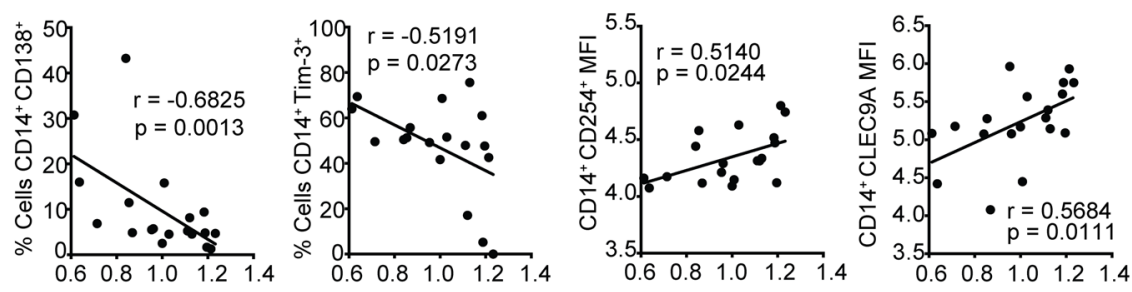

T2 Lesion Load (ratio pre/post $12 \mathrm{~m}$ IFN- $\beta$ treatment)
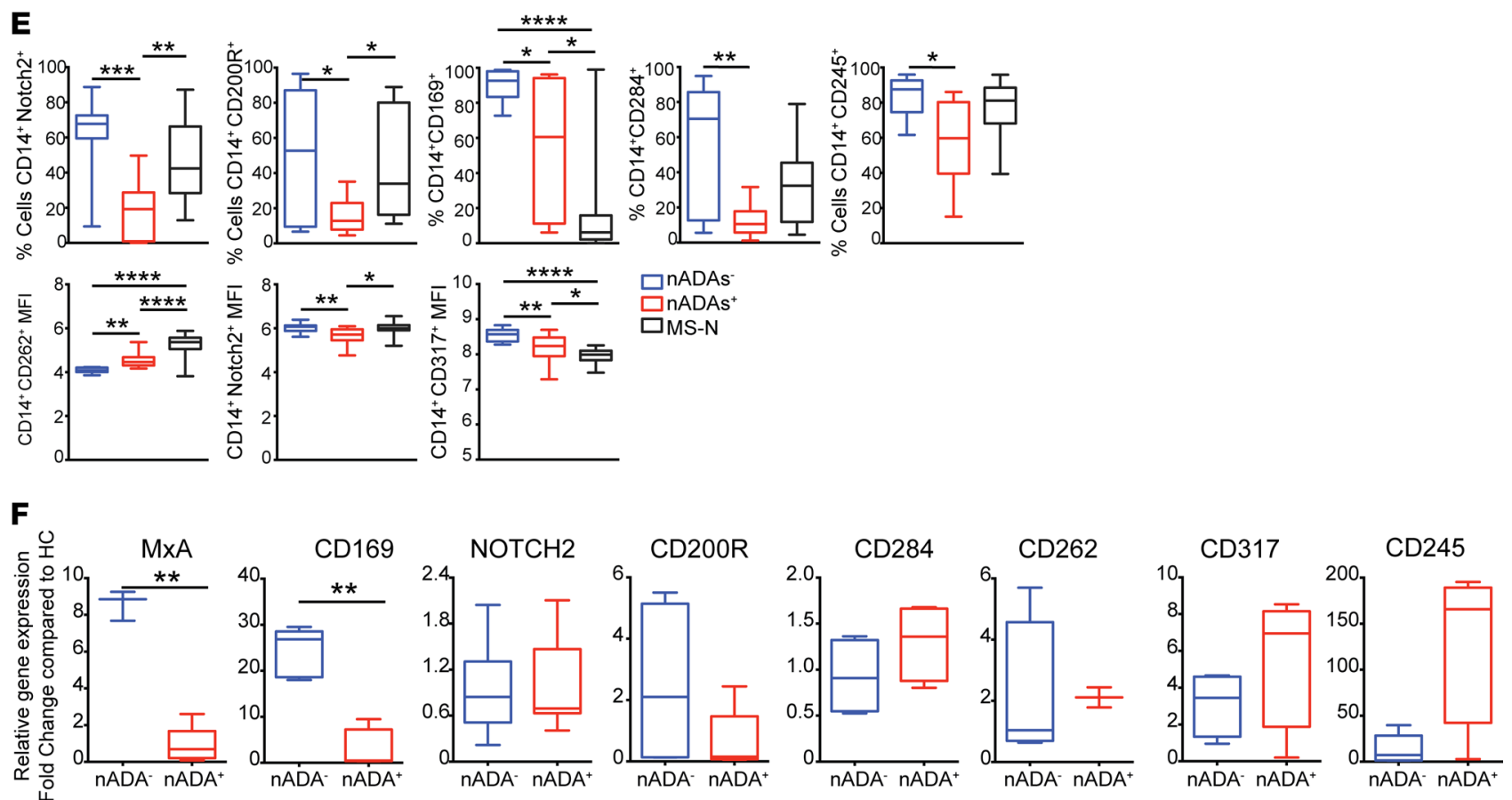

Figure 2. Using LegendScreen to identify the immune signature associated with neutralizing antidrug antibodies (nADA) in patients with multiple sclerosis (MS). (A) Peripheral blood mononuclear cells (PBMCs) from MS patients treated with IFN- $\beta$ for at least 12 months (MS-T; Table 1, discovery cohort) and who were either $\operatorname{nADA}^{-}(n=12)$ or $\operatorname{nADA}^{+}(n=16)$ were analyzed using LegendScreen. Volcano plots comparing fold change (Log 2$)$ in marker expression against the significance of marker expression between nADA- and nADA ${ }^{+}$MS patients in monocytes, B cells, and T cells (Log ${ }_{10} P$ value). Only markers listed in Supplemental Table 1 were included in the analysis. Horizontal dashed line represents $P$ value threshold $(P=0.05)$, vertical dashed lines represent fold change in marker expression (dashed fold change, 0 ; dotted blue lines fold change, \pm 1 ). Markers significantly different between $\mathrm{nADA}^{-} / \mathrm{nADA}^{+}$after $0.20 \mathrm{FDR}$ and with a Log fold change $\geq \pm 0.4$ were selected for further analysis (Supplemental Table 2 ). (B) Summary flow chart of the screening strategy used to identify the nADA-associated phenotypic signature. (C and $\mathbf{D}$ ) Correlation of differentially expressed markers (DEMs) selected in A and Supplemental Table 2 with disease progression assessed by MRI measurements T1 (C) and T2 (D). Changes in brain lesions detected by comparing MRI scanning at the time of PBMC isolation to MRI scans performed 12 months before sampling. Spearman's correlation coefficient ( $r$ ) (see 
also Supplemental Table 3). (E) DEM expression in treatment-naive (MS-N; $n=15), n A D A^{-}(n=9)$, and nADA+ $(n=11)$ IFN- $\beta$-treated (MS-T) patients. Box and whisker plots showing expression of NOTCH2, CD200R, CD169, CD284, CD245, CD262, and CD317 on monocytes that passed inclusion criteria in Table 2. One-way ANOVA with Tukey's correction for multiple comparisons, ${ }^{*} P \leq 0.05$, ${ }^{* *} P \leq 0.01,{ }^{* * *} P \leq 0.001$, ${ }^{* * *} P \leq 0.0001$. See Supplemental Figure 3 for analysis of excluded markers. (F) Transcriptional regulation of the selected nADA-associated markers (Supplemental Table 4). mRNA was isolated from FACS-sorted CD14+ monocytes from nADA $(n \geq 3)$ and nADA $(n \geq 3)$ MS-T patients and analyzed for CD169, NOTCH2, CD200R, CD284, CD262, CD317, and CD245 and for IFN- $\beta$-responsive gene MXA as a control analyzed by qPCR relative to cyclophilin or GAPDH. Fold change compared with HCs shown. Twotailed $t$ tests, ${ }^{* *} P=0.01$. Box plots show the median and 25 th and 75 th percentiles; whiskers represent minimum and maximum values.

with the effect of IFN- $\beta$ and removed from further analysis. Thus using high-throughput flow cytometry, an immune signature associated with nADA development in a cohort of IFN- $\beta$-treated MS patients and exclusively expressed on $\mathrm{CD} 14^{+}$monocytes was pinpointed (Supplemental Table 4).

To evaluate whether altered surface expression of the nADA-associated markers was related to changes in gene expression, quantitative PCR (qPCR) analysis was performed on RNA isolated from FACS-sorted $\mathrm{CD}_{14}{ }^{+}$monocytes from $\mathrm{nADA}{ }^{-}$and $\mathrm{nADA}^{+} \mathrm{MS}$ patients (Figure $2 \mathrm{~F}$ ). No difference in gene expression was found in the NOTCH2, CD200R, CD284, CD262, CD317, and CD245 between nADA ${ }^{-}$and nADA ${ }^{+} \mathrm{MS}^{-T}$ patients, suggesting that changes in cell surface expression may be mediated by posttranscriptional regulation mechanisms. However, MxA and CD169/SIGLEC1 (genes known to respond to IFN- $\beta$; refs. 14, 20) were significantly upregulated in monocytes from nADA ${ }^{-}$compared with nADA ${ }^{+} \mathrm{MS}$ patients, showing that the presence of nADA blocks the effect of IFN- $\beta$ therapy in these cells. Thus, the results suggest that changes in cell surface expression did not correlate with gene transcription and RNA detection and may be mediated by posttranscriptional regulation mechanisms $(21,22)$.

Reduced NOTCH2 surface expression on monocytes was associated with $n A D A$ development in MS patients treated with IFN- $\beta$. To validate the identified nADA-associated signature, a second independent cross-sectional cohort comprising 23 relapsing-remitting MS (RRMS)/clinically isolated syndrome (CIS) patients treated for 12 months with IFN- $\beta$ and tested for the presence of nADA at sampling (Table 1, validation cohort; Figure 3A) was analyzed for the expression of the markers listed in Supplemental Table 4. This analysis confirmed that NOTCH2, CD245, CD169, CD262, and CD317 were associated with nADA development; however, CD200R and CD284 were not found to be differentially expressed in this cohort and were excluded from the signature. Therefore, 5 markers expressed on monocytes were confirmed to be associated with the presence of nADAs in MS patients treated for over 12 months with IFN- $\beta$ (Table 3 ).

Finally, the expression of the remaining markers was examined in PBMCs collected from a prospective cohort of 18 newly diagnosed RRMS/CIS patients before the first injection of IFN- $\beta$ and followed up for 12 months (Table 1, prospective cohort). Marker expression was evaluated blindly and stratified according to the presence of nADA assessed after 12 months of IFN- $\beta$ treatment (nADA prospective ${ }^{+}\left[\mathrm{nADA}_{\mathrm{p}}^{+}\right]$or $\mathrm{nADA}_{\mathrm{p}}^{-}$). Strikingly, only NOTCH2 was significantly reduced on the surface of $\mathrm{CD} 14^{+}$monocytes from untreated MS patients that went on to develop nADA after 12 months of IFN- $\beta$ therapy (Figure 3B). IFN- $\beta$ treatment did not influence the expression of NOTCH2 on monocytes overtime, nor did the type of treatment or the nADA induction and titre (Supplemental Figure 4, A-D, and Supplemental Table 6). Therefore, these data suggest that reduced detection of NOTCH2 on $\mathrm{CD} 14^{+}$monocytes could be a potential biomarker to predict nADA development in MS-N patients.

Combining NOTCH2 surface expression and monocyte subset frequency predicted nADA development in IFN- $\beta$ treated $M S$ patients. To gain insight into the pathophysiological significance of monocyte NOTCH2 expression associated with nADA induction, we analyzed the expression of NOTCH2 in functional monocyte subsets. Human monocytes can be subdivided into 3 subsets based on the expression of CD14 and CD16 in peripheral blood; namely, classical $\left(\mathrm{CD} 14^{+} \mathrm{CD} 16^{-}\right)$, intermediate $\left(\mathrm{CD} 14^{+} \mathrm{CD} 16^{+}\right)$, and nonclassical $\left(\mathrm{CD} 14^{\mathrm{lo}} \mathrm{CD} 16^{+}\right)$ monocytes $(23,24)$. NOTCH2 signaling is known to regulate monocyte cell fate by driving monocyte differentiation from the mainly phagocytic classical subset toward more proinflammatory, intermediate, and nonclassical phenotypes (25). Therefore, we assessed subset frequency in both the validation and prospective MS patient cohorts $(26,27)$ (Table 1 and Figure $4 \mathrm{~A}$ for gating strategy). MS-T patients with established nADA $\left(\mathrm{nADA}{ }^{+}\right)$had significantly elevated numbers of proinflammatory nonclassical monocytes $\left(\mathrm{CD} 14^{\mathrm{lo}} \mathrm{CD} 16^{+}\right)$ and reduced classical monocytes $\left(\mathrm{CD} 14^{+} \mathrm{CD} 16^{-}\right)$compared with $\mathrm{nADA}^{-} \mathrm{MS}-\mathrm{T}$ patients (Figure $4, \mathrm{~B}$ and C). The increase in the nonclassical monocyte subset in $\mathrm{nADA}^{+}$patients was not associated with disease activity (Supplemental Figure 5). Patients from the prospective cohort recruited before IFN- $\beta$ administration and classified as $n \mathrm{ADA}^{+}$after 12 months of IFN- $\beta$ therapy $\left(\mathrm{nADA}_{\mathrm{p}}^{+}\right)$had significantly elevated intermediate 
Table 2. Strategy used to identify differentially expressed markers (DEMs) associated with the effect of IFN- $\beta$-treatment only.

\begin{tabular}{|c|c|c|}
\hline \multicolumn{3}{|c|}{ DEM selection criteria } \\
\hline $\begin{array}{l}\text { nADA- vs MS-N } \\
\text { and } \\
\text { nADA+ vs MS-N }\end{array}$ & $\begin{array}{c}P=<0.05 \\
\text { NS }\end{array}$ & Excluded \\
\hline $\begin{array}{l}\text { nADA- vs MS-N } \\
\text { and } \\
\text { nADA+ vs MS-N } \\
\text { OR }\end{array}$ & $\begin{array}{l}P=<0.05 \\
P=<0.05\end{array}$ & \multirow{3}{*}{ Included } \\
\hline $\begin{array}{l}\text { nADA- vs MS-N } \\
\text { and } \\
\text { nADA+ vs MS-N } \\
\text { OR }\end{array}$ & $\begin{array}{l}\text { NS } \\
\text { NS }\end{array}$ & \\
\hline $\begin{array}{l}\text { nADA- vs MS-N } \\
\text { and } \\
\text { nADA+ vs MS-N }\end{array}$ & $\begin{array}{c}\text { NS } \\
P=<0.05\end{array}$ & \\
\hline $\begin{array}{l}\text { Strategy used to identify differentially ex } \\
\text { (PBMCs) from multiple sclerosis (MS) pati } \\
\text { positive (nADA } ; n=16 \text { ) were analysed usi } \\
\text { nADA }{ }^{-} \text {and MS-N MS patients (Table } 1 \text {, dis }\end{array}$ & $\begin{array}{l}\text { h the effect } \\
\text { months and } \\
\text { See Figure } 2\end{array}$ & $\begin{array}{l}\text { al blood mononuclear cells } \\
\text { their expression in } \mathrm{AADA}^{+} \text {, }\end{array}$ \\
\hline
\end{tabular}

rather than nonclassical monocytes compared with $\mathrm{nADA}_{\mathrm{p}}^{-}$patients (Figure 4, D and E). Correspondingly, NOTCH2 expression was reduced in all monocyte subsets in $\mathrm{nADA}^{+}$and $\mathrm{nADA}_{\mathrm{p}}^{+}$patients compared with $\mathrm{nADA}^{-}$and $\mathrm{nADA}_{\mathrm{p}}^{-}$patients, respectively (Figure 4, F and G). This suggested that reduced NOTCH2 expression and altered monocyte subset frequencies could be a feature in both patients who had already developed nADA and in those patients that would go on to develop nADA after IFN- $\beta$ treatment. Scatterplot 3-dimensional (3-D) and unsupervised hierarchical cluster analysis using intermediate and nonclassical monocyte frequency and total monocyte NOTCH2 expression levels clustered MS-N patients from the prospective cohort into 2 distinct groups; 1 group that went on to develop $\mathrm{nADA}\left(\mathrm{nADA}_{\mathrm{p}}^{+}\right)$and 1 group that did not $\left(\mathrm{nADA}_{\mathrm{p}}^{-}\right.$) (Figure 4, $\mathrm{H}$ and I), suggesting that these parameters could be used to stratify patients before they receive treatment to identify those at greatest risk of nADA development. In support of this, receiver operating characteristic (ROC) curves constructed using a predictor built using NOTCH2 expression and nonclassical monocyte subset frequency in the discovery cohort and applied to predict ADA status in the validation cohort show an AUC value of 0.88 , indicating a higher predictive power than the NOTCH2 expression alone (NOTCH2 mean fluroescence intensisty [MFI] and NOTCH2 percentage AUC values 0.85 and 0.70 , respectively) (Figure 4J). Moreover, repeating the same approach using patients from the prospective cohort to build the predictor model (a logistic regression model) and applying the predictor model to treatment-naive MS patients whose baseline samples were analyzed using the LegendScreen platform (Table 1), we identified that $\mathrm{NOTCH} 2 \mathrm{MFI}$ could discriminate $\mathrm{nADA}_{\mathrm{p}}{ }^{-}$and $\mathrm{nADA}_{\mathrm{p}}{ }^{+}$patients before initiation of therapy (AUC = 0.75) (Supplemental Figure 6).

Serum factors drive increased NOTCH2 activation in $n A D A^{+}$and $n A D A_{p}^{+} M S$ patients. Reduced detection of surface NOTCH2 in monocytes (Figure 4, B-G) could be due to increased binding to NOTCH2 ligands, triggering the expression of specific NOTCH2 target genes - including HES1 (hairy and enhancer of split-1) and DTX1 (Deltex1) (28-30). Indeed, NOTCH2-responsive genes HES1 and DTX1 were upregulated (Figure 5, A and B), and the mRNA and protein expression of proinflammatory IL- 6 were increased in monocytes from $\mathrm{nADA}^{+}$and $\mathrm{nADA}_{\mathrm{p}}^{+} \mathrm{MS}$ patients compared with $\mathrm{nADA}^{-}$and $\mathrm{nADA}_{\mathrm{p}}^{-}$patients, respectively (Figure 5, C-G). Thus, in MS patients who had developed or who went on to develop nADA, NOTCH2 appeared to be preferentially activated, potentially driving proinflammatory cytokine production and monocyte differentiation. Confirming this, when HC monocytes were cultured in the presence of serum from $\mathrm{nADA}^{+} / \mathrm{nADA}^{-} \mathrm{MS}$ patients, only nADA ${ }^{+}$serum induced increased HES1 and DTX1 expression, an effect that was blocked by inhibiting NOTCH2 activation using a specific inhibitor of $\gamma$-secretase (N-[N-(3, 5-difluorophenacetyl)-1-alanyl]-S-phenylglycine t-butyl ester; DAPT) (Figure 5, H-J). Moreover, the induction of $\mathrm{NOTCH} 2$ target genes by $\mathrm{nADA}^{+}$patient serum was associated with reduced transcription 
A
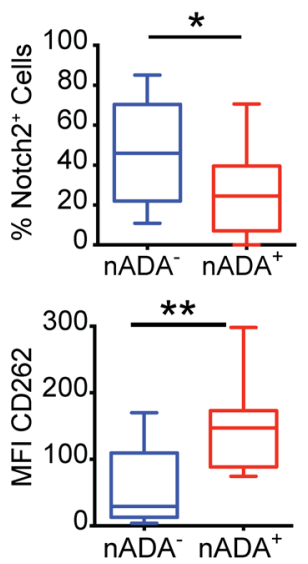
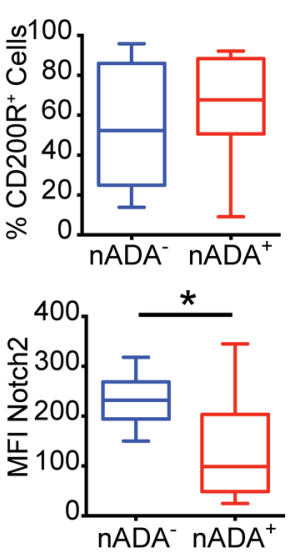
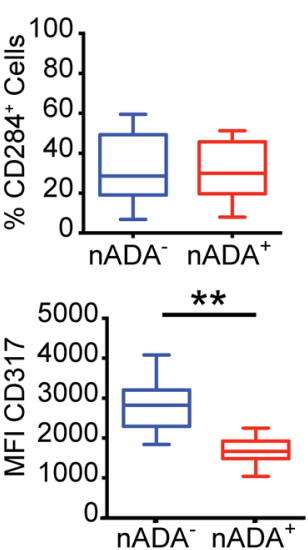
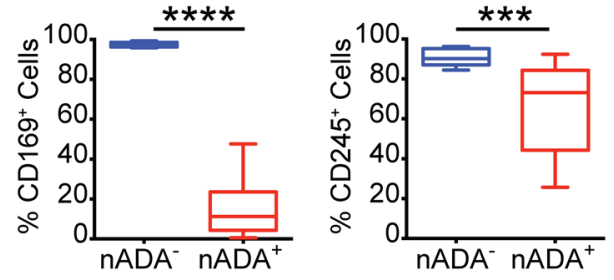

B
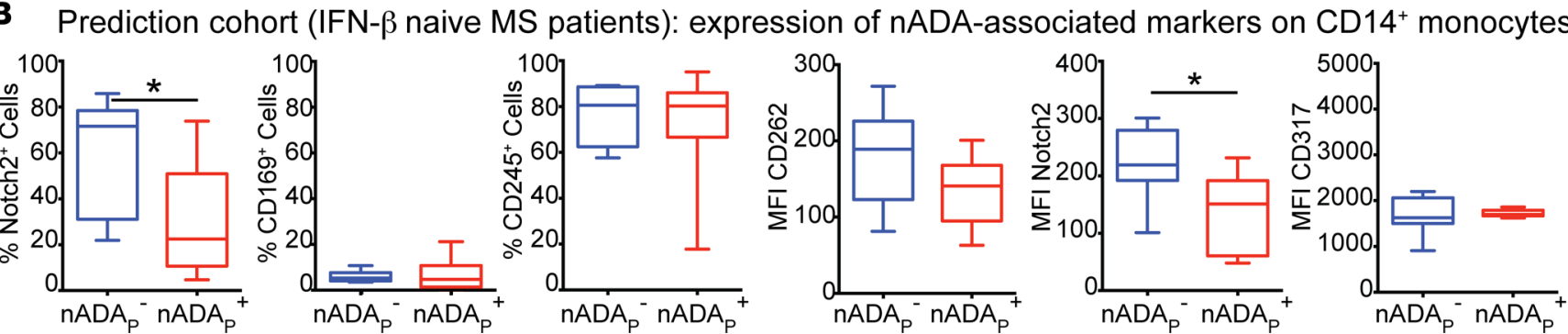

Figure 3. NOTCH2 expression on monocytes could be a predictor of neutralizing antidrug antibody (nADA) development in IFN- $\beta$ naive multiple sclersosis (MS) patients. (A) Peripheral blood mononuclear cells (PBMCs) from an independent cohort of IFN- $\beta$-treated (MS-T) patients either $n A D A^{-}(n=10)$ or $n A D A^{+}$ $(n=13)$, treated with IFN- $\beta$ for at least 12 months (validation cohort, Table 1) were analyzed for the expression of NOTCH2, CD200R, CD284, CD169, CD245, CD262, and CD317 on CD14+ monocytes using flow cytometry. Two-tailed unpaired $t$ tests, ${ }^{*} P \leq 0.05,{ }^{*} P \leq 0.01,{ }^{* * *} P \leq 0.001,{ }^{* * *} P \leq 0.0001$. (B) PBMCs from treatment-naive (MS-N) patients $(n=18)$ taken before the first injection of IFN- $\beta$ (prediction cohort, Table 1 ) were analyzed for the expression of NOTCH2, CD169, CD245, CD262, and CD317 on CD14+ monocytes using flow cytometry. The expression of nADA-associated markers was stratified according to the presence of nADA in the same patients after they had been treated for 12 months with IFN- $\beta$; nADA prospective-positive ( $n A D A{ }_{p}{ }^{+}$) $(n=11)$ and $n A D A_{p}{ }^{-}(n$ = 7). Mann Whitney $U$ test, ${ }^{*} P<0.05$. Box plots show the median and 25 th and 75 th percentiles; whiskers represent minimum and maximum values.

of $C D 163$, a marker of classical monocytes, and increased expression of genes associated with inflammation and nonclassical monocyte subsets including STAT1 (Figure 5K and Supplemental Figure 7) (31, 32).

Finally, the mechanism of monocyte NOTCH2 activation was assessed in a coculture experiment (Figure 5L). Induction of HES1 by $\mathrm{nADA}^{+}$serum was only observed in monocytes cocultured with $\mathrm{T}$ cells - not when monocytes were cocultured with B cells or in monocyte-only cultures (Figure 5L). Therefore, the data suggest that patients with nADA had serum factors that triggered the activation of NOTCH2 on monocytes in a non-cell intrinsic manner and that NOTCH2 activation could initiate the differentiation of proinflammatory monocyte subsets and provide an environment favoring ADA development (Figure 6). Thus, patients who had this proinflammatory signature prior to their first treatment with IFN- $\beta$ could be predisposed to develop nADA.

\section{Discussion}

Systems biology approaches are used increasingly to identify molecular signatures that orchestrate complex immunological processes. Such studies support the concept that multiplex measurements, including gene expression, high-density analysis of cell populations, and cellular and serological responses, can predict the type and magnitude of human immune responses (33). Here, high-throughput flow cytometry was used to identify a unique phenotypic signature associated with the development of neutralizing antibodies to IFN- $\beta$ in patients with MS. Notably, reduced expression of NOTCH2 on monocytes was associated with nADA development in patients with MS treated with IFN- $\beta$ and was also identified as a potential predictive marker of nADA development in patients who were drug naive using a prospective patient cohort. Serum factors in patients with nADA triggered NOTCH2 activation and subsequently reduced 
Table 3. Validated list of nADA-associated markers

\begin{tabular}{lcccc}
\hline Cell subsets & Markers & P value & Average expression nADA $^{-}$ & Average expression nADA $^{+}$ \\
& NOTCH2 & 0.000387312 & 60.485 & 26.1367 \\
CD14 ${ }^{+}$monocytes (\% positive) & CD169 & 0.0110785 & 91.7758 & 60.1069 \\
& CD245 & 0.0166616 & 85.3675 & 66.0625 \\
& CD262 & $6.162782 \mathrm{e}-005$ & 4.09066 & 4.54363 \\
CD14 $^{+}$monocytes (MFI) & NOTCH2 & 0.00216778 & 6.032 & 5.66506 \\
& CD317 & 0.00238299 & 8.54727 & 8.19584
\end{tabular}

detection of surface NOTCH2 in monocytes. This process drove the differentiation of proinflammatory monocyte subsets, an effect that required $\mathrm{T}$ cells but not B cells. Thus, this work has identified a potentially novel mechanism influencing the development of immunogenicity to biopharmaceuticals and a potential biomarker to predict antidrug responses. The role of NOTCH2 and monocyte subsets in the development of $\mathrm{nADA}$ in the wider context of other biopharmaceutical drugs and in different diseases is, at present, under investigation (34-36).

NOTCH2 signaling is a highly conserved pathway involved in cell fate decisions, proliferation, and survival (37). Mammalian cells have 4 NOTCH receptors (NOTCH-1 to -4) and 5 NOTCH ligands (Delta-like-1, -3 , and -4 and Jagged-1 and -2). NOTCH/NOTCH-ligand binding induces $\gamma$-secretase-mediated cleavage and shedding of the NOTCH extracellular domain, activation of the NOTCH signaling pathway, and induction of NOTCH target genes, including HES1 and DTX1 (28, 30, 38). Our data revealed that NOTCH2 gene expression was similar between $\mathrm{nADA}^{-}$and $\mathrm{nADA}^{+} \mathrm{MS}$ patients, while membrane expression was reduced in the latter, most likely indicating an association between NOTCH pathway activation and nADA induction. Alternatively, posttranslational modification of the NOTCH extracellular domain, which has been reported to control the strength of NOTCH-pathway signaling events and the selective interaction between NOTCH and NOTCH ligands, could also account for the reduced detection of membrane NOTCH2 in $\mathrm{nADA}^{+}$MS patients $(39,40)$. However, our finding that $\mathrm{CD} 14^{+}$monocytes isolated from $\mathrm{nADA}^{+} \mathrm{MS}$ patients had significantly increased expression of both HES1 and DTX1 genes compared with both $\mathrm{nADA}^{-} \mathrm{MS}$ patients and HCs supported the hypothesis that the reduced expression of membrane NOTCH2 was due to increased receptor activation. NOTCH target gene transcription can also be stimulated by TLR-2 and TLR- 4 activation with LPS and the fungal analog zymosan, respectively, independently of NOTCH receptor activation $(41,42)$. However, our observation that $\mathrm{nADA}^{+}$, but not $\mathrm{nADA}^{-}$, patient serum could upregulate HES1 and DTX1 transcription in HC monocytes and that this could be inhibited by pretreating PBMCs with the NOTCH-specific $\gamma$-secretase inhibitor DAPT demonstrated the direct association between NOTCH receptor, NOTCH target gene activation, and the loss of tolerance to IFN- $\beta$ in MS. The fact that the surface expression of all 4 mammalian NOTCH receptors on $\mathrm{CD} 14^{+}$monocytes was measured in the discovery cohort and only the surface expression of NOTCH2 was found to be significantly different between the $\mathrm{nADA}^{+}$and $\mathrm{nADA}^{-}$MS patients groups suggested that NOTCH2 activation in $\mathrm{CD} 14^{+}$monocytes was associated with the generation of ADA against IFN- $\beta$ in MS.

Human monocytes can be subdivided into 3 subsets based on the expression of CD14 and CD16 and characterized by a specific gene and microRNA (miRNA) signature $(23,31)$. The classical $\left(\mathrm{CD} 14^{+} \mathrm{CD} 16^{-}\right)$ monocyte subset constitutes $85 \%-90 \%$ of the circulating monocyte pool, whereas the remaining $10 \%-15 \%$ consist of intermediate $\left(\mathrm{CD} 14^{+} \mathrm{CD} 16^{+}\right)$and nonclassical $\left(\mathrm{CD} 14^{1 \circ} \mathrm{CD} 16^{+}\right)$monocytes $(31,43)$. In humans, monocyte precursors differentiate into classical monocytes in the BM and further polarize toward intermediate and nonclassical monocytes in the peripheral blood (24). While the function of monocyte subsets is still debated, recent evidence suggests that classical monocytes are mainly phagocytic and not inflammatory, while nonclassical monocytes produce inflammatory cytokines and exhibit proprieties of antigen presentation, and the intermediate subset may be transitional with both inflammatory and phagocytic functions (44). Indeed, NOTCH2 activation regulates the in vivo differentiation of $\mathrm{Ly} 6 \mathrm{C}^{\text {lo }}$ monocytes, the murine equivalent of the human nonclassical monocytes, from peripheral LyC ${ }^{\text {hi }}$ (murine classical monocytes) monocytes (25). This suggests that the observed upregulation of NOTCH2 signaling in $\mathrm{nADA}^{+}$ patients could be responsible for the induction of nonclassical monocytes in this patient subset. We show 
A From live/single
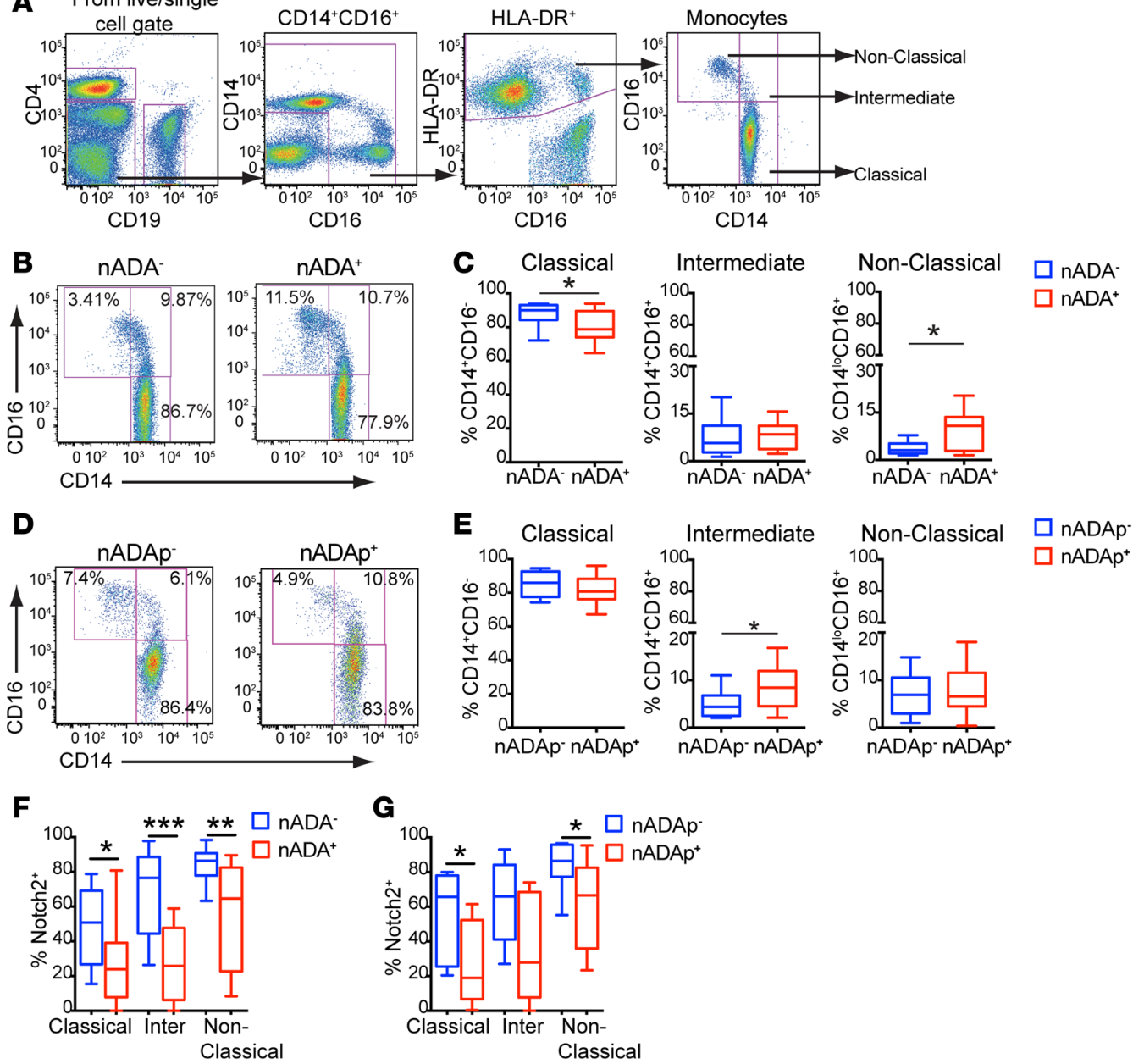

\section{H}

Naive MS: NADA $_{\mathrm{p}}^{-} \quad$ Naive MS: nADA ${ }^{+}$

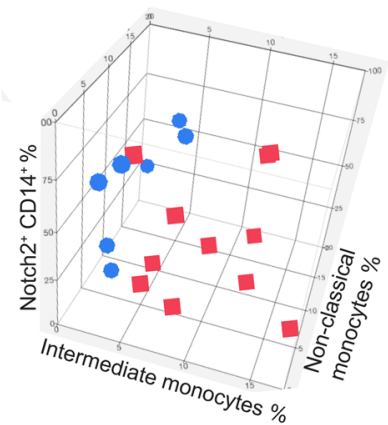

I

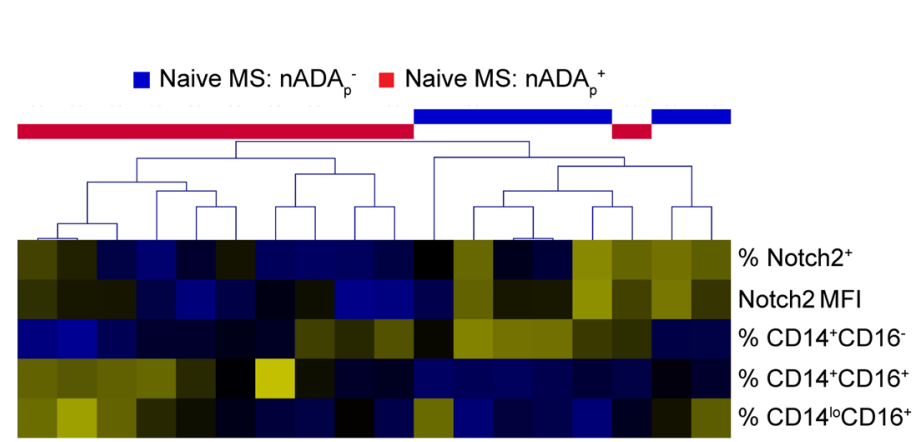

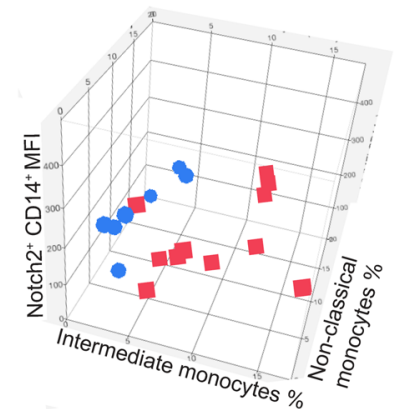

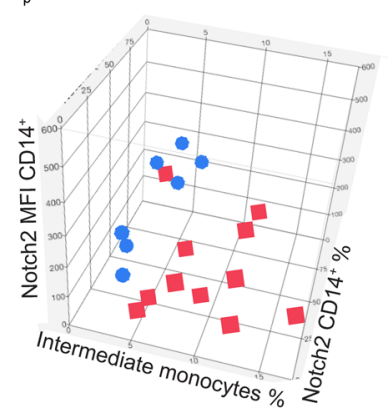

J

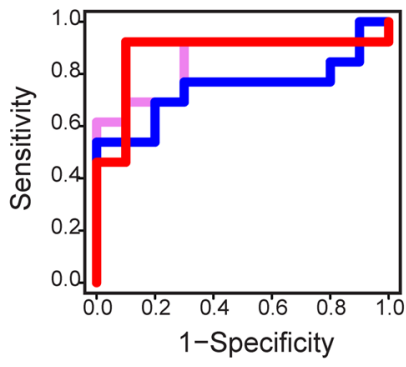

- Notch-2 MFI, AUC $=0.85$

Non-classical monocytes, $A \cup C=0.70$

- Notch-2 MFI + non-classic mono, AUC $=0.88$

Figure 4. Combining monocyte subset frequency and NOTCH2 expression as a predictive marker of neutralizing antidrug antibody (nADA) development. (A) Flow cytometry gating strategy for monocyte subsets based on CD14 and CD16 expression; classical (CD14+CD16-), intermediate (CD14+CD16 ${ }^{+}$), and nonclassical $\left(C_{14}^{\circ}{ }^{\circ} \mathrm{CD}_{16}{ }^{+}\right)(27)$. Frequency of classical, intermediate, and nonclassical monocytes in nADA ${ }^{+}(n=13)$ or nADA $(n=10)$ IFN- $\beta$-treated (MS-T) patients 
and $\mathrm{nADA}_{\mathrm{p}}{ }^{+}(n=11)$ and $\mathrm{nADA}{ }_{\mathrm{p}}^{-}(n=7)$ treatment-naive (MS-N) patients. Representative dotplots (B and $\left.\mathbf{D}\right)$ and cumulative data (C and $\left.\mathbf{E}\right)$. Mann Whitney $U$ test, ${ }^{*} P \leq 0.05$. (F and $\left.\mathbf{G}\right)$ Expression of NOTCH2 (\%) on the surface of monocyte subsets from nADA ${ }^{+} / \mathrm{nADA}^{-} \mathrm{MS}^{-} \mathrm{T}$ and nADA ${ }_{\mathrm{p}} / \mathrm{nADA} \mathrm{p}_{\mathrm{p}}^{-} \mathrm{MS}-\mathrm{N}$ patients. Mann Whitney $U$ test, ${ }^{*} P \leq 0.05$, ${ }^{*} P \leq 0.01$, ${ }^{* *} P \leq 0.001$. Box plots show the median and 25 th and 75 th percentiles; whiskers represent minimum and maximum values. (H) Three-dimensional scatterplots combining intermediate and nonclassical subset frequencies and CD14 ${ }^{+}$monocyte NOTCH2 expression (\% and mean fluroescence intensisty [MFI]) from nADA ${ }_{p}^{-}(n=7)$ and nADA ${ }_{p}^{+}(n=11)$ MS-N patients. Generated using JMP version 12.0.1 software (www.jmp.com). (I) Unsupervised hierarchical clustering of patients as in $\mathbf{H}$. (J) Receiver operation characteristic (ROC) curves detailing the predictive value of NOTCH2 expression (MFI) and of the frequency of nonclassical monocytes alone (pink and blue lines, respectively) and of the 2 variables combined (red line).

that IFN- $\beta$-treated $n \mathrm{ADA}^{+} \mathrm{MS}$ patients had significantly elevated numbers of proinflammatory nonclassical monocytes $\left(\mathrm{CD} 14^{\mathrm{lo}} \mathrm{CD} 16^{+}\right)$and reduced classical monocytes $\left(\mathrm{CD} 14^{+} \mathrm{CD} 16^{-}\right)$compared with $\mathrm{nADA}^{-}$ patients. NOTCH2 surface expression was significantly reduced in classical and intermediate monocyte subsets in $\mathrm{nADA}^{+}$compared with $\mathrm{nADA}^{-} \mathrm{MS}$ patients, suggesting that the activation of the NOTCH2 pathway in these 2 subsets could induce the accumulation of nonclassical monocytes in $\mathrm{nADA}^{+}$patients.

Patients in the prospective cohort that went on to develop nADA ( $\mathrm{ADA}_{\mathrm{p}}^{+}$) had reduced surface NOTCH2 on classical monocytes and an increased frequency of intermediate monocytes compared with patients that did not go on to develop nADA ( $\mathrm{nADA}_{\mathrm{p}}^{-}$) even before they received the first dose of IFN- $\beta$. Monocytes from $\mathrm{nADA}^{+}$and $\mathrm{nADA}_{\mathrm{p}}^{+}$patients were also characterized by increased expression of the proinflammatory cytokine IL-6 compared with nADA ${ }^{-}$and $\mathrm{nADA}_{\mathrm{p}}^{-}$, respectively. Taken together, these data support a link between NOTCH2 activation and the differentiation of monocytes from classical (mainly phagocytic) to proinflammatory intermediate and nonclassical subsets $(24,25)$.

The activation and recruitment of peripheral blood monocytes to the CNS is a hallmark of active disease associated with ongoing demyelination and axonal injury, underlining the role of myeloid cells in the disease pathophysiology $(8,45)$. An association between MS disease activity and circulating myeloid cell activation and proinflammatory cytokine release has been demonstrated previously (46-50). Higher frequencies of IL- 6 and IL-12 secreting monocytes have been reported in MS patients compared with healthy donors (46). Moreover, the frequency of IL-12 releasing monocytes has been shown to correlate with the presence of gadolinium-enhancing MRI in patients (47). However, the evidence surrounding perturbations in circulating monocyte subset frequencies in MS patients has been inconsistent $(48,49)$. Previously, IFN- $\beta$ treatment has been shown to either induce accumulation of circulating $\mathrm{CD}_{1} 6^{+}$intermediate and nonclassical monocytes (49) or to increase the intermediate subset frequency at the expense of nonclassical monocytes in MS (50). Here, we observed increased CD16 expression on CD14 ${ }^{+}$monocytes from MS-T compared with untreated patients, suggesting that IFN- $\beta$ directly induced CD16 expression on monocytes. Moreover, the frequency of intermediate monocytes between $\mathrm{nADA}^{+}$and $\mathrm{nADA}^{-} \mathrm{MS}$ patients was comparable, suggesting that the reduced frequency of nonclassical $\left(\mathrm{CD} 14^{\mathrm{lo}} \mathrm{CD} 16^{+}\right)$subsets in the $\mathrm{nADA}^{+}$patients was not due to the effect of IFN- $\beta$.

It is known that the $E$. coli-derived nonglycosylated IFN- $\beta-1 b$ is more immunogenic than the fully glycosylated IFN- $\beta-1$ a produced in mammalian CHO cells (51). The majority of $\mathrm{nADA}^{+}$patients in our cohorts were treated with the more immunogenetic form of IFN- $\beta-1 b$; this did not allow us to clearly discriminate the effect of the different preparations on the expression of NOTCH2. Furthermore, although NOTCH2 expression on monocytes was a good predictor of immunogenicity against IFN- $\beta$, our study was not powered enough to establish a clear cut-point to use in routine clinical practice to identify patients likely to develop nADA. Further studies are warranted to identify such cut-point values.

The stepwise approach used here was designed to identify DEMs associated with nADA, excluding any marker associated with disease progression (e.g., TIM-3) (52) or response to IFN- $\beta$ therapy (e.g., CD274, CD123, CD129, ect.) (53-55). IFN- $\beta$-neutralizing antibodies only partially explain IFN- $\beta$ nonresponse in MS (56). Qualitative and quantitative differences in the molecular response to the drug, genetic variants in IFN- $\beta$ receptors, or signaling components and perturbations of monocytes and the innate immune system are all reported to be associated with reduced response to therapy (57-60). While - for the purposes of this study - markers associated with disease progression or response to IFN- $\beta$ therapy were excluded, they could play an important role in MS pathogenesis and/or response to treatment and will be the subject of further investigations.

In summary, we show for the first time to our knowledge that reduced surface expression of NOTCH2 and increased NOTCH intracellular signaling in monocytes was associated with increased frequency of nonclassical $\left(\mathrm{CD} 14^{\mathrm{lo}} \mathrm{CD} 16^{+}\right)$monocytes and nADA induction in IFN- $\beta$-treated MS patients. This signature was identified in both cross-sectional and prospective patient cohorts and supports the hypothesis 
A



B

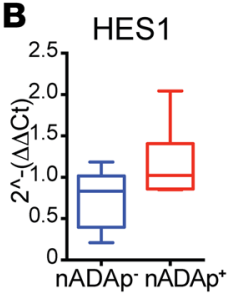

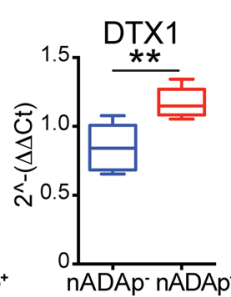

C

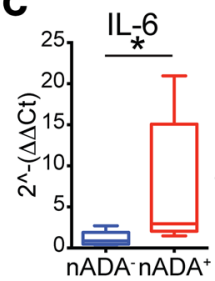

D

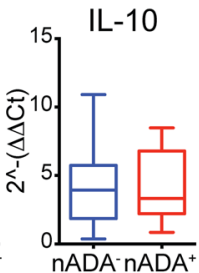

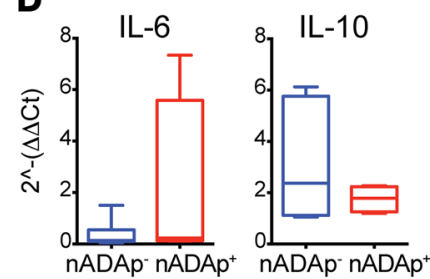

E

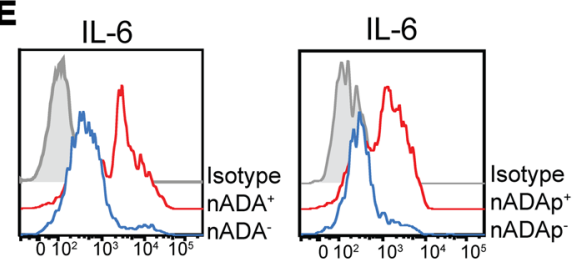

$\mathbf{F}$

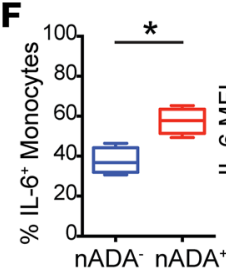

G

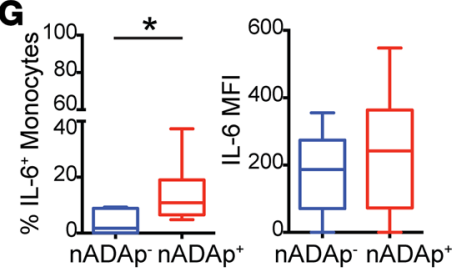

H

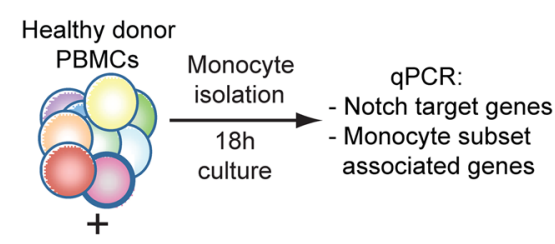

Medium with $10 \%$

human serum

$\left(\mathrm{nADA} ; \mathrm{nADA}^{+}\right)$

$+/-\gamma$-secreatase inhibitor

(DAPT)
I

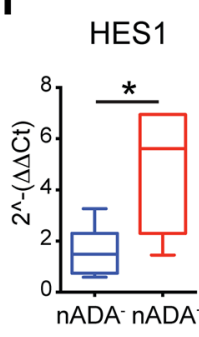

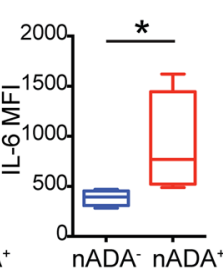

J

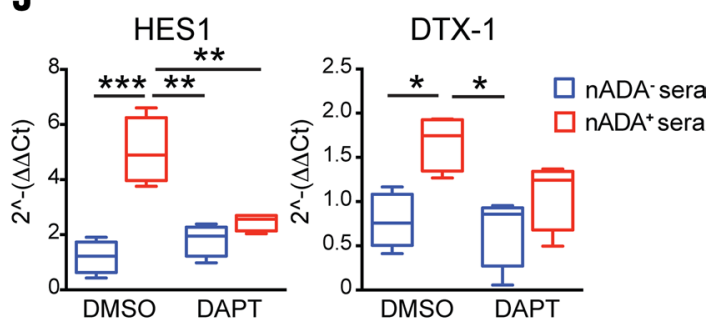

$\mathbf{K}$

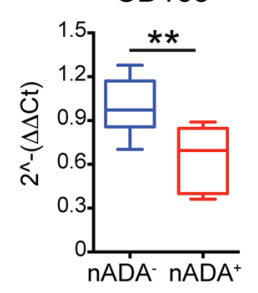

STAT-1

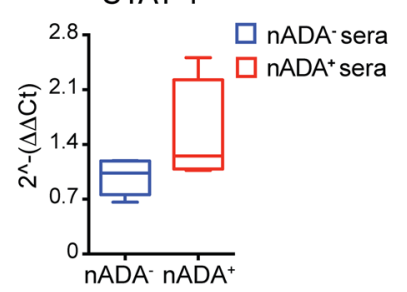

$\mathbf{L}$

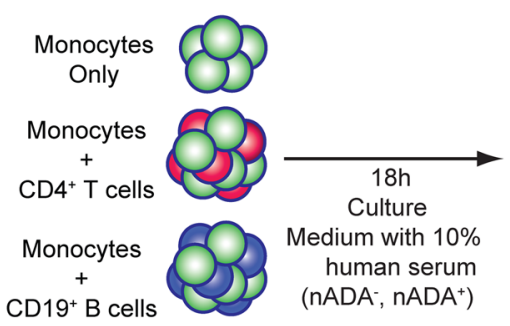

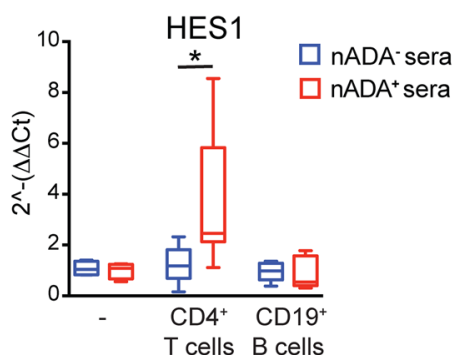

Figure 5. Serum factors drive increased NOTCH2 activation in neutralizing antidrug antibody-positive (nADA ${ }^{+}$multiple sclerosis (MS) patients. (A-D) mRNA isolated from ex vivo FACS-sorted CD14+ monocytes from healthy donors (HCs, $n=8), \mathrm{nADA}^{-}(n=8) / \mathrm{nADA}+(n=8)$ IFN- $\beta$-treated (MS-T) patients and nADA ${ }_{p}^{+}(n=6) / \mathrm{nADA}_{\mathrm{p}}{ }^{-}(n=6)$ treatment-naive (MS-N) patients. NOTCH target genes HES1 and DTX1 (A and B) and cytokine genes IL6 and IL10 (C and $\mathbf{D}$ ) were analyzed by qPCR relative to cyclophilin. Results are expressed as fold change from HCs. Mann Whitney $U$ test, ${ }^{*} P \leq 0.05,{ }^{* *} P \leq 0.01$. (E-G) Ex vivo peripheral blood mononuclear cells (PBMCs) from nADA ${ }^{-}(n=4) / n \mathrm{nDA}^{+}(n=4) \mathrm{MS}^{-T}$ patients and nADA ${ }_{\mathrm{p}}{ }(n=$ $6) / \mathrm{nADA}_{\mathrm{p}}^{-}(n=6)$ MS-N patients were cultured for 6 hours in presence LPS $(10 \mu \mathrm{g} / \mathrm{ml})$. PMA $(50 \mathrm{ng} / \mathrm{ml})$, ionomycin $(250 \mathrm{ng} / \mathrm{ml})$, and Brefeldin A $(1 \mu \mathrm{g} /$ $\mathrm{ml}$ ) were added to the cultures for the last 2 hours before harvesting. Intracellular IL-6 expression was evaluated by flow cytometry. (E) Representative histograms and cumulative data (percentage positive and mean fluorescence intensity; MFI) showing IL-6 expression in monocytes from (F) MS-T and (G) MS-N patients. Mann Whitney $U$ test, ${ }^{*} P<0.05$. (H) Experimental strategy for in vitro experiments. HC PBMCs were cultured with $10 \%$ serum from MS-T nADA+ or nADA- patients. Following 18-hour culture, mRNA was isolated from purified monocytes for qPCR analysis. (I) Cumulative expression of NOTCH target genes HES1 and DTX1 relative to cyclophilin A in nADA- $(n=6)$ or nADA $(n=5)$ MS-T patients evaluated by qPCR. Mann Whitney $U$ test, ${ }^{*} P<0.05$. (J) PBMCs were precultured for 16 hours $\pm N$-[N-(3,5-difluorophenacetyl-L-alanyl)]-S-phenylglycine $t$-butyl ester (DAPT) or vehicle (DMSO) before 18 -hour culture with $10 \%$ serum from nADA $/ \mathrm{nADA}^{-}$patients $(n=4)$. Cumulative expression of $H E S 1$ and $D T X 1$ relative to cyclophilin A. Mann Whitney $U$ test, ${ }^{*} P<0.05$, ${ }^{* *} P \leq 0.01$, ${ }^{* * *} P \leq 0.001$. (K) Cumulative expression of genes previously reported to be associated to classical (CD163) or nonclassical (STAT1) monocyte subsets (32). (L) HES1 expression in sorted CD14+ monocyte cultures and cocultures of CD14+ monocytes and CD19+ $B$ cells or CD14 monocytes and CD4+ T cells (1:1 ratio) after culture in presence of serum from MS-T nADA ${ }^{+}$or $n A D A^{-}$ patients $(n=7)$. Mann Whitney $U$ test. ${ }^{*} P<0.05$. Box plots show the median and 25 th and 75 th percentiles; whiskers represent minimum and maximum values. 
A

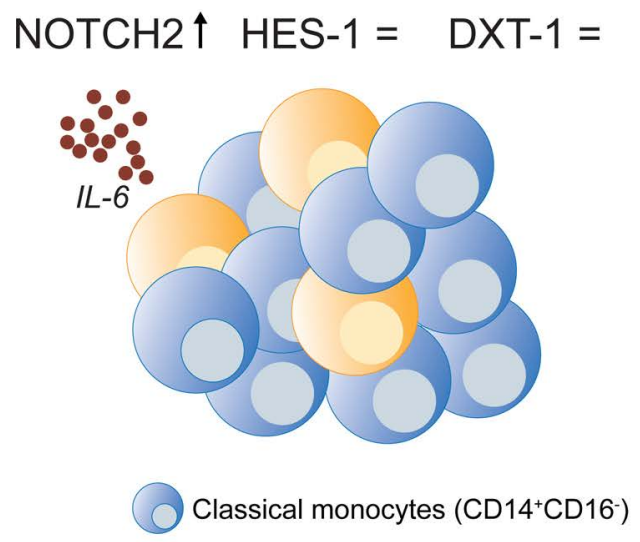

B

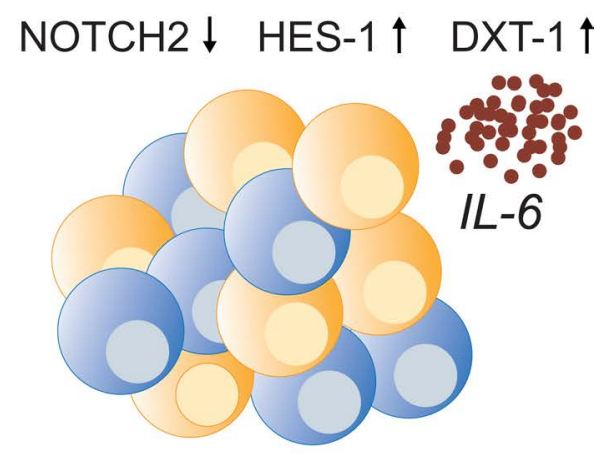

Intermediate/Non-classical

monocytes $\left(\mathrm{CD} 14^{+/ / 0} \mathrm{CD} 16^{+/ h i}\right)$

Figure 6. Increased NOTCH2 signaling could predispose multiple sclerosis (MS) patients to immunogenicity. (A) MS patients treated with IFN- $\beta$ who had elevated monocyte NOTCH2 expression and low levels of NOTCH2 activation were less likely to develop nADA compared with (B) MS patients with reduced monocyte NOTCH2 expression and increased monocyte NOTCH2 activation. Reduced surface expression and increased expression of NOTCH2 target genes HES1 and DTX1 were associated with increased intermediate/nonclassical monocyte subset frequencies and increased monocyte IL-6 production. MS patients with increased NOTCH2 activation and increased proinflammatory monocyte subset frequencies were predisposed to develop nADA following treatment with IFN- $\beta$.

that a preexisting variation in the immune profile and cellular make-up of MS patients may contribute to immunogenicity against IFN- $\beta$ and could be used as a biomarker to predict response to biopharmaceuticals in a clinical setting.

\section{Methods}

Patient cohorts. Patients were diagnosed with RRMS or CIS according to the revised McDonald criteria (61). Three cohorts were recruited and summarized in Table 1. Briefly, PBMCs and serum were collected from a discovery cohort, either as MS-N or treated with IFN- $\beta$ for at least 12 months (MS-T). A validation cohort of MS-T patients and a prospective cohort were recruited as part of the Anti-Biopharmaceutical Immunization: prediction and analysis of clinical relevance to minimize the RISK consortium (ABIRISK consortium; www.abirisk.eu/), with samples collected from patients before their first treatment with IFN- $\beta$ and after 12 months of treatment. Patients included in the study were treated with both $E$. coli-derived IFN- $\beta$-1b (Betaferon/Extavia, $n=33$ ) or mammalian-derived IFN- $\beta-1$ a (Avonex, $n=8$; Rebif, $n=43$ ) products. PBMCs and serum were collected from age-, sex-, ethnicity-, disease duration-, and smoking status-matched HC. In order to reduce drug interference with the expression of immune cell markers, all samples were collected 14 hours after the last administration of IFN- $\beta$. PBMCs were isolated using density gradient centrifugation and cryopreserved in liquid nitrogen until use. Serum samples were used to test for the presence of $\mathrm{nADA}$.

MRI acquisition and lesion volumetric assessment. All MRI scans analyzed in the study were performed on the same scanner (1.5 T Gyroscan; Philips Medical Systems) in the Department of Radiodiagnostics at General University Hospital in Prague with the same protocol. The standardized protocol consisted of 2 sequences: fluid-attenuated inversion recovery (FLAIR) and T1-weighted 3-dimensional fast field echo (T1-WI/FFE 3D). Contiguous slices covering the whole brain were acquired with the following parameters. FLAIR sequence: time to echo (TE), $140 \mathrm{~ms}$; time to repetition (TR), 11,000 $\mathrm{ms}$; inversion time (TI), 2,600 ms; matrix size, $256 \times 181$; flip angle (FA), $90^{\circ}$; slice thickness (THK), 1.5/0 mm (with no gaps); field of view (FOV), $256 \mathrm{~mm}$; and T1-WI/FFE 3D (TE/TR, 5/25 ms; FA, $30^{\circ}$; matrix size, $256 \times 256$; THK, 1.0/0 mm; FOV, $256 \mathrm{~mm}$ ). Volumetric assessment was performed in the Department of Radiodiagnostic, First Faculty of Medicine and General University Hospital in Prague, as reported (62). Changes in lesions size were evaluated calculating the ratio between measurements taken at 12 months duration.

$n A D A$ detection. Serum from MS-N and MS-T patients were tested for nADA using a luciferase-based bioassay (LUC) (63). 
LegendScreen screening. PBMCs were analyzed using LegendScreen Human Cell Screening Kits (BioLegend; http://www.biolegend.com/legendscreen), according to the manufacturer's protocol with the following modifications. Briefly, $3 \times 10^{7}\left(\sim 9 \times 10^{4}\right.$ per antibody) PBMCs were labeled with a 1:5 dilution of each PE-labeled $\mathrm{mAb}$ targeting 332 surface antigen and 10 isotype controls, making up the LegendScreen panel. After 30 minutes of incubation at $4^{\circ} \mathrm{C}$, cells were washed and incubated with CD4-V500 (RPA-T4; BD Biosciences), CD19-APC-Cy7 (SJ25C1; BD Biosciences), and CD14-APC (61D3; eBioscience) to identify CD4+ T cell, B cell, and monocyte subsets for 30 minutes at $4^{\circ} \mathrm{C}$. After final washing, cells were fixed before acquisition using the FACSVerse flow cytometer equipped with FACSuite software (Becton Dickinson), and 25,000-40,000 events were acquired per marker. For all samples, aggregates and dead cells were excluded by forward- and side-scatter gating; fluorescence minus one (FMO) controls were used to control for specificity of surface antigen staining. Data were analyzed by manual gating using FlowJo 8.8.7 software (TreeStar Inc.). Percentage of PE-positive cells and PE median MFI were collected for each of the 332 markers screened.

Mixture model for LegendScreen marker selection. The markers expressed by the different cell subpopulations were selected using a 2-component Gaussian mixture model on the log-scale (intensities were highly positively skewed) performed using Rmixmod in $\mathrm{R}$ version 3.3.0 and fitted to the data using a stochastic expectation-maximization (EM) algorithm. The mixture modeling was then followed by a 4 class clustering: (a) a unimodal-negative class containing the markers with 2 negative modes; (b) a unimodal-positive class with 2 positive modes; (c) a bimodal class with 1 negative mode and 1 positive mode; and (d) a bimodal class with 2 positive modes. The threshold values were chosen based on several gold-standard negative markers (confirmed in the literature to be negative for each cell subset examined) (64) (Supplemental Table 5 and Supplemental Figure 2, A and B).

Monocyte subset staining. PBMCs $\left(1 \times 10^{7}\right)$ were labeled with HLA-DR-APC-Cy7 (clone L243), CD4BV785 (clone RPA-T4), CD19-BV711 (clone SJ25C1), CD14-BV510 (clone 63D3), CD16-Alexa Fluor 647 (clone 3G8), CXCR1-Fitc (clone 8F1/CXCR1), CD192-PerCp-Cy5.5 (clone K036C2), CD206-BV421 (clone 15-2), and CD68-PE-Cy7 (clone Y1/82A) (all from BioLegend) for 30 minutes at $4^{\circ} \mathrm{C}$. Live cells were detected using the Live/Dead Blue Fixable Stain (Invitrogen), followed by singlet gates. FMOs and isotype controls were used to gate the cell subsets of interest (27). At least 5,000 CD14 ${ }^{+}$cells per sample were acquired using a LSR Fortessa X20 cytometer equipped with FACS Diva software (Becton Dickinson) and analyzed using FlowJo software, as stated previously.

Intracellular cytokines. PBMCs were stimulated with $10 \mu \mathrm{g} / \mathrm{ml}$ LPS (MilliporeSigma) for 6 hours. PMA $(50 \mathrm{ng} / \mathrm{ml})$, ionomycin $(250 \mathrm{ng} / \mathrm{ml})$, and Brefeldin A $(1 \mu \mathrm{g} / \mathrm{ml})$ were added to the cultures for the last 2 hours before harvesting. After culture, cells were stained with CD4-BV785 (clone RPA-T4), CD16-APCCy7 (clone 3G8), CD19-BV711 (clone SJ25C1), and HLA-DR-APC-Cy7 (clone L243) (all from BioLegend) and CD14-APC (clone 61D3; eBioscience), washed twice in PBS fixed with IC fixation buffer (eBioscience). IL-6 staining was performed in permeabilization buffer (eBioscience) at $4^{\circ} \mathrm{C}$ for 40 minutes (specific antibody dilution 1:25).

Monocyte, T cell, and B cell isolation. Purified cell populations were obtained using negative bead isolation (Stemcell Technologies) according to manufacturer's instructions or FACS sorting.

Cell culture. HC PBMCs rested for 16 hours in complete media (RPMI 1640 [MilliporeSigma] supplemented with 10\% heat-inactivated FBS [Labtech International Ltd.], $100 \mathrm{U} / \mathrm{ml}$ penicillin, and $100 \mu \mathrm{g} /$ $\mathrm{ml}$ streptomycin [MilliporeSigma]) were washed 2 times in cold PBS and seeded in 24-well plates $(5 \times$ $10^{6}$ cells/well) in RPMI enriched with $10 \%$ patient (either $\mathrm{nADA}^{-}$or $\mathrm{nADA}^{+}$) or $\mathrm{HC}$ sera. After 18 hours, cells were harvested and CD14 ${ }^{+}$monocytes positively isolated (EasySep; Stemcell Technologies) according to manufacturer's instructions. In some experiments, $30 \mu \mathrm{M}$ of the $\gamma$-secretase inhibitor $N$-[ $N$-(3,5-difluorophenacetyl-L-alanyl)]-S-phenylglycine $t$-butyl ester (DAPT) or equal volume of DMSO (all from MilliporeSigma) were added to the PBMCs during the resting stage and during the 18-hour culture in the presence of serum.

Gene expression assay. Total RNA was extracted from sorted $\mathrm{CD} 14^{+}$cells using PicoPure RNA Isolation Kit (Invitrogen), according to manufacturer's instructions. RNA was reverse-transcribed to cDNA with the iScript Reverse Transcription Supermix (Bio-Rad), and gene expression was measured by qPCR. PCR primers used were as follows: actin (forward, 5'-AGATGACCCAGATCATGTTTGAG-3'; reverse, 5'-AGGTCCAGACGCAGGATG-3'), cyclophilin A (forward, 5'-GCATACGGGTCCTGGCATCTTGTCC-3'; reverse, 5'-ATGGTGATCTTCTTGCTGGTCTTGC-3'), IL6 (forward, 5'-CCAGGAGCCCAGCTATGAAC-3'; reverse, 5'-CCCAGGGAGAAGGCAACTG-3'), TNFA (forward, 5'-TCTTCTCGAACCCCGAGTGA-3'; 
reverse, 5'-CCTCTGATGGCACCACCAG-3'), CD200R (forward, 5'-CCATCGTGGGATTCATTTGGT-3'; reverse, 5'-GGCTGCATTTCATCCTCCTC-3'), or IL10, CD169, CD262, CD245/MYO18A, CD317, IRF9, $M X 1$, and NOTCH2 QuantiTect primers (QIAGEN). GAPDH and CD284/TLR4 expression was evaluated using TaqMan Gene Expression Assays (Thermo Fisher scientific). The relative expression level of specific transcripts was normalized with respect to the internal standard ( $\beta$-actin, cyclophilin A, or GAPDH). Relative expression was calculated against healthy control via the $\Delta \Delta \mathrm{Ct}$ method.

Statistics. Statistical analysis was performed with the Prism Software (GraphPad), JMP version 12.0.1 software (www.jmp.com), and the R software package Rmixmod (65). The significance of differences among experimental groups was assessed by 2-tailed Student's $t$ test, Mann Whitney $U$ test or 1-way ANOVA followed by Tukey's post hoc test. Data was presented using box and whisker plots; the box shows the median and 25th and 75th percentiles, and the whiskers represent minimum and maximum values. Correlations were assessed with Spearman's correlation coefficient. $P>0.05$ was considered significant; ${ }^{*} P<$ $0.05,{ }^{* *} P<0.01,{ }^{* *} P<0.001$.

In order to assess the capacity of Notch2 and monocyte subset frequency to predict nADA development in MS-N patients, predictors were constructed on the discovery cohort using univariate and multivariate logistic models with NOTCH2 MFI, NOTCH2 percentage-positive monocytes, and frequency of each monocyte subset as explanatory variables. The models with the best fit to the data measured as lowest Akaike Information Criterion (AIC) were applied on the validation cohort to predict the ADA status and build ROC curves, on which the AUC was calculated using the R package ROCR.

Study approval. Ethical approval was obtained for this study from the ethics committee of the University College London Hospitals National Health Service Trust, London, United Kingdom (15/SW/0109); Medical Ethics Committee of the General University Hospital in Prague (125/12, Evropský grant 1.LF UK-CAGEKID); Ethikkommission der Fakultät für Medizin der Technischen Universität München, München, Germany (project no. 335/13); Ethikkommission Nordwest- und Zentralschweiz, Basel, Switzerland (project no. 305/13); and Ethikkommission der Medizinischen Universität Innsbruck, Innsbruck, Austria (UN2013-0040_LEK). Written informed consent was obtained from all participants in accordance with the Declaration of Helsinki principles.

\section{Author contributions}

Research study designed by ECJ, C. Mauri, MA, PD, AFH, C. Mbogning, SH, and VM; experiments conducted by MA; experimental data acquired by MA; data analyzed by MA, ECJ, C. Mbogning, SH, $\mathrm{PN}, \mathrm{PD}, \mathrm{PB}, \mathrm{AFH}$, and $\mathrm{ZB}$; patient recruitment and assessment contributed by $\mathrm{PN}, \mathrm{AFH}, \mathrm{FD}, \mathrm{KM}, \mathrm{BH}$, RLPLG, CS, and NF; nADA screening and analysis performed by PEHJ, PC, CW, KI, AFH, and FD; manuscript written by ECJ and MA; and manuscript reviewed by C. Mauri, SH, PN, PD, PB, AFH, FD, KW, EKH, PEHJ, KM, ZB, VM, and the ABIRISK consortium.

\section{Acknowledgments}

The authors are grateful to Hans-Peter Hartung (University of Düsseldorf, Germany), Bernd Kieseier (University of Düsseldorf, Germany), Xavier Montalban (Hospital Universitari Vall d'Hebron, Spain), Manuel Comabella (Hospital Universitari Vall d'Hebron, Spain), Dorothea Buck (Technische Universität München, Germany), Marc Pallardy (INSERM UMR 996, France), Malin Ryner (Karolinska Institutet, Sweden), Sophie Tourdot (INSERM UMR 996, France), Dan Sikkema (GlaxoSmithKline, USA), and Sebastian Spindeldreher (Novartis, Switzerland) for patient cohort recruitment,samples collection and/or allocation, and management of the ABIRISK project (www.abirisk.eu). The study was performed as part of the Innovative Medicines Initiative Joint Undertaking under grant agreement no. 115303, resources of which are composed of financial contribution from the European Union's Seventh Framework Program (FP7/2007-2013) and European Federation of Pharmaceutical Industries and Associations (EFPIA) in kind contribution.

Address correspondence to: Elizabeth Jury, Marsilio Adriani, or Claudia Mauri, University College London, Center for Rheumatology Research, Rayne Building, 5 University Street, London WC1E 6JF, United Kingdom. Phone: 44.0.2031082161; Email: e.jury@ucl.ac.uk (L. Jury). Phone: 44.0.2031082154; Email: a_marsilio@hotmail.com (M. Adriani). Phone: 44.0.2031082155; Email: c.mauri@ucl.ac.uk (C. Mauri). 
1. Brownlee WJ, Hardy TA, Fazekas F, Miller DH. Diagnosis of multiple sclerosis: progress and challenges. Lancet. 2017;389(10076):1336-1346.

2. Sawcer S, Franklin RJ, Ban M. Multiple sclerosis genetics. Lancet Neurol. 2014;13(7):700-709.

3. Ajami B, Bennett JL, Krieger C, McNagny KM, Rossi FM. Infiltrating monocytes trigger EAE progression, but do not contribute to the resident microglia pool. Nat Neurosci. 2011;14(9):1142-1149.

4. Yamasaki R, et al. Differential roles of microglia and monocytes in the inflamed central nervous system. J Exp Med. 2014;211(8):1533-1549.

5. Dendrou CA, Fugger L, Friese MA. Immunopathology of multiple sclerosis. Nat Rev Immunol. 2015;15(9):545-558.

6. Nikbin B, Bonab MM, Khosravi F, Talebian F. Role of B cells in pathogenesis of multiple sclerosis. Int Rev Neurobiol. 2007;79:13-42.

7. Lopez-Diego RS, Weiner HL. Novel therapeutic strategies for multiple sclerosis--a multifaceted adversary. Nat Rev Drug Discov. 2008;7(11):909-925.

8. Mishra MK, Yong VW. Myeloid cells - targets of medication in multiple sclerosis. Nat Rev Neurol. 2016;12(9):539-551.

9. [No authors listed]. Neutralizing antibodies during treatment of multiple sclerosis with interferon beta-1b: experience during the first three years. The IFNB Multiple Sclerosis Study Group and the University of British Columbia MS/MRI Analysis Group. Neurology. 1996;47(4):889-894.

10. Jacobs LD, et al. Intramuscular interferon beta-1a for disease progression in relapsing multiple sclerosis. The Multiple Sclerosis Collaborative Research Group (MSCRG). Ann Neurol. 1996;39(3):285-294.

11. Fisher E, et al. Eight-year follow-up study of brain atrophy in patients with MS. Neurology. 2002;59(9):1412-1420.

12. Applebee A, Panitch H. Early stage and long term treatment of multiple sclerosis with interferon-beta. Biologics. 2009;3:257-271.

13. Bachelet D, et al. Occurrence of Anti-Drug Antibodies against Interferon-Beta and Natalizumab in Multiple Sclerosis: A Collaborative Cohort Analysis. PLoS One. 2016;11(11):e0162752.

14. Sominanda A, Hillert J, Fogdell-Hahn A. In vivo bioactivity of interferon-beta in multiple sclerosis patients with neutralising antibodies is titre-dependent. J Neurol Neurosurg Psychiatry. 2008;79(1):57-62.

15. Neumann TA, Foote M. Megakaryocyte growth and development factor (MGDF): an Mpl ligand and cytokine that regulates thrombopoiesis. Cytokines Cell Mol Ther. 2000;6(1):47-56.

16. Sorensen PS. Neutralizing antibodies against interferon-Beta. Ther Adv Neurol Disord. 2008;1(2):125-141.

17. Schellekens H. The immunogenicity of biopharmaceuticals. Neurology. 2003;61(9 Suppl 5):S11-S12.

18. Link J, et al. Human leukocyte antigen genes and interferon beta preparations influence risk of developing neutralizing antidrug antibodies in multiple sclerosis. PLoS One. 2014;9(3):e90479.

19. Wattjes MP et al. Evidence-based guidelines: MAGNIMS consensus guidelines on the use of MRI in multiple sclerosis-establishing disease prognosis and monitoring patients. Nat Rev Neurol. 2015;11(10):597-606.

20. Malhotra S, et al. Search for specific biomarkers of IFN $\beta$ bioactivity in patients with multiple sclerosis. PLoS One. 2011;6(8):e23634.

21. Graessel A, et al. A Combined Omics Approach to Generate the Surface Atlas of Human Naive CD4+ T Cells during Early T-Cell Receptor Activation. Mol Cell Proteomics. 2015;14(8):2085-2102.

22. Rugg-Gunn PJ, et al. Cell-surface proteomics identifies lineage-specific markers of embryo-derived stem cells. Dev Cell. 2012;22(4):887-901.

23. Ginhoux F, Jung S. Monocytes and macrophages: developmental pathways and tissue homeostasis. Nat Rev Immunol. 2014;14(6):392-404

24. Patel AA, et al. The fate and lifespan of human monocyte subsets in steady state and systemic inflammation. J Exp Med. 2017;214(7):1913-1923.

25. Gamrekelashvili J, et al. Regulation of monocyte cell fate by blood vessels mediated by Notch signalling. Nat Commun. 2016;7:12597.

26. Auffray C, Sieweke MH, Geissmann F. Blood monocytes: development, heterogeneity, and relationship with dendritic cells. Annu Rev Immunol. 2009;27:669-692.

27. Abeles RD, et al. CD14, CD16 and HLA-DR reliably identifies human monocytes and their subsets in the context of pathologically reduced HLA-DR expression by CD14(hi) /CD16(neg) monocytes: Expansion of CD14(hi) /CD16(pos) and contraction of CD14(lo) /CD16(pos) monocytes in acute liver failure. Cytometry A. 2012;81(10):823-834.

28. Ilagan MX, Kopan R. SnapShot: notch signaling pathway. Cell. 2007;128(6):1246.

29. Kopan R, Ilagan MX. The canonical Notch signaling pathway: unfolding the activation mechanism. Cell. 2009;137(2):216-233.

30. Tsao PN, et al. Lipopolysaccharide-induced Notch signaling activation through JNK-dependent pathway regulates inflammatory response. J Biomed Sci. 2011;18:56.

31. Wong KL, et al. Gene expression profiling reveals the defining features of the classical, intermediate, and nonclassical human monocyte subsets. Blood. 2011;118(5):e16-e31.

32. Gren ST, Rasmussen TB, Janciauskiene S, Håkansson K, Gerwien JG, Grip O. A Single-Cell Gene-Expression Profile Reveals Inter-Cellular Heterogeneity within Human Monocyte Subsets. PLoS ONE. 2015;10(12):e0144351.

33. Li S, et al. Molecular signatures of antibody responses derived from a systems biology study of five human vaccines. Nat Immunol. 2014;15(2):195-204

34. Walsh G. Biopharmaceutical benchmarks 2010. Nat Biotechnol. 2010;28(9):917-924.

35. Walsh G. Biopharmaceutical benchmarks 2014. Nat Biotechnol. 2014;32(10):992-1000.

36. Wolfe RM, Ang DC. Biologic Therapies for Autoimmune and Connective Tissue Diseases. Immunol Allergy Clin North Am. 2017;37(2):283-299.

37. Bray SJ. Notch signalling: a simple pathway becomes complex. Nat Rev Mol Cell Biol. 2006;7(9):678-689.

38. Saito T, et al. Notch2 is preferentially expressed in mature B cells and indispensable for marginal zone B lineage development. Immunity. 2003;18(5):675-685.

39. Benedito R, et al. The notch ligands Dl14 and Jagged1 have opposing effects on angiogenesis. Cell. 2009;137(6):1124-1135

40. Van de Walle I, et al. Jagged2 acts as a Delta-like Notch ligand during early hematopoietic cell fate decisions. Blood. 
2011;117(17):4449-4459.

41. Alvarez Y, et al. Notch- and transducin-like enhancer of split (TLE)-dependent histone deacetylation explain interleukin 12 (IL-12) p70 inhibition by zymosan. J Biol Chem. 2011;286(19):16583-16595.

42. Hu X, et al. Integrated regulation of Toll-like receptor responses by Notch and interferon-gamma pathways. Immunity. 2008;29(5):691-703.

43. Passlick B, Flieger D, Ziegler-Heitbrock HW. Identification and characterization of a novel monocyte subpopulation in human peripheral blood. Blood. 1989;74(7):2527-2534.

44. Mukherjee R, Kanti Barman P, Kumar Thatoi P, Tripathy R, Kumar Das B, Ravindran B. Non-Classical monocytes display inflammatory features: Validation in Sepsis and Systemic Lupus Erythematous. Sci Rep. 2015;5:13886.

45. Lucchinetti C, Brück W, Parisi J, Scheithauer B, Rodriguez M, Lassmann H. Heterogeneity of multiple sclerosis lesions: implications for the pathogenesis of demyelination. Ann Neurol. 2000;47(6):707-717.

46. Kouwenhoven M, Teleshova N, Ozenci V, Press R, Link H. Monocytes in multiple sclerosis: phenotype and cytokine profile. J Neuroimmunol. 2001;112(1-2):197-205.

47. Makhlouf K, Weiner HL, Khoury SJ. Increased percentage of IL-12+ monocytes in the blood correlates with the presence of active MRI lesions in MS. J Neuroimmunol. 2001;119(1):145-149.

48. Chuluundorj D, Harding SA, Abernethy D, La Flamme AC. Expansion and preferential activation of the CD14(+)CD16(+) monocyte subset during multiple sclerosis. Immunol Cell Biol. 2014;92(6):509-517.

49. Waschbisch A, et al. Pivotal Role for CD16+ Monocytes in Immune Surveillance of the Central Nervous System. J Immunol. 2016;196(4):1558-1567.

50. Then Bergh F, Dayyani F, Ziegler-Heitbrock L. Impact of type-I-interferon on monocyte subsets and their differentiation to dendritic cells. An in vivo and ex vivo study in multiple sclerosis patients treated with interferon-beta. J Neuroimmunol. 2004;146(1-2):176-188.

51. Sominanda A, Rot U, Suoniemi M, Deisenhammer F, Hillert J, Fogdell-Hahn A. Interferon beta preparations for the treatment of multiple sclerosis patients differ in neutralizing antibody seroprevalence and immunogenicity. Mult Scler. 2007;13(2):208-214

52. Li Z, Ju Z, Frieri M. The T-cell immunoglobulin and mucin domain (Tim) gene family in asthma, allergy, and autoimmunity. Allergy Asthma Proc. 2013;34(1):e21-e26.

53. Trabattoni $\mathrm{D}$, et al. Costimulatory pathways in multiple sclerosis: distinctive expression of PD-1 and PD-L1 in patients with different patterns of disease. J Immunol. 2009;183(8):4984-4993.

54. Huang YM, Adikari S, Båve U, Sanna A, Alm G. Multiple sclerosis: interferon-beta induces CD123(+)BDCA2- dendritic cells that produce IL-6 and IL-10 and have no enhanced type I interferon production. J Neuroimmunol. 2005;158(1-2):204-212.

55. Ding X, Cao F, Cui L, Ciric B, Zhang GX, Rostami A. IL-9 signaling affects central nervous system resident cells during inflammatory stimuli. Exp Mol Pathol. 2015;99(3):570-574.

56. Bertolotto A, Gilli F. Interferon-beta responders and non-responders. A biological approach. Neurol Sci. 2008;29 Suppl 2:S216-S217.

57. Rani MR, et al. Heterogeneous, longitudinally stable molecular signatures in response to interferon-beta. Ann N Y Acad Sci. 2009;1182:58-68

58. Rudick RA, et al. Excessive biologic response to IFN $\beta$ is associated with poor treatment response in patients with multiple sclerosis. PLoS One. 2011;6(5):e19262.

59. Comabella $\mathrm{M}$, et al. A type I interferon signature in monocytes is associated with poor response to interferon-beta in multiple sclerosis. Brain. 2009;132(Pt 12):3353-3365.

60 . Hegen $\mathrm{H}$, et al. Cytokine profiles show heterogeneity of interferon- $\beta$ response in multiple sclerosis patients. Neurol Neuroimmunol Neuroinflamm. 2016;3(2):e202.

61. Polman CH, et al. Diagnostic criteria for multiple sclerosis: 2010 revisions to the McDonald criteria. Ann Neurol. 2011;69(2):292-302.

62. Uher T, et al. A Novel Semiautomated Pipeline to Measure Brain Atrophy and Lesion Burden in Multiple Sclerosis: A LongTerm Comparative Study. J Neuroimaging. 2017;27(6):620-629.

63. Hermanrud C, et al. Development and validation of cell-based luciferase reporter gene assays for measuring neutralizing antidrug antibodies against interferon beta. J Immunol Methods. 2016;430:1-9.

64. BD Biosciences. Human and Mouse CD Marker Handbook. San Diego, California, USA: BD Biosciences; 2010. https://www. bdbiosciences.com/documents/cd_marker_handbook.pdf. Accessed May 17, 2018.

65. Lebret R, et al. Rmixmod: The R Package of the Model-Based Unsupervised, Supervised, and Semi-Supervised Classification Mixmod Library. J Stat Software. 2015;67(6):1-29. 